\begin{tabular}{|c|c|c|c|c|c|}
\hline MUNIBE Antropologia-Arkeologia & $n^{\circ} 67$ & $235-252$ & DONOSTIA & 2016 & ISSN 1132-2217 • eISSN 2172-4555 \\
\hline
\end{tabular}

\title{
Patrones de aprovisionamiento de sílex de las comunidades superopaleolíticas del Pirineo occidental: el "coste" como medida de análisis a partir de los SIG
}

\author{
Flint procurement patterns of Upper Paleolithic communities \\ of the western Pyrenees: \\ "cost" as a measure of analysis using GIS
}

\begin{abstract}
PALABRAS CLAVES: Cost Distance Analysis, Unidades de Coste, difusión del sílex, lógica difusa, Paleolítico superior. GAKO-HITZAK: Cost Distance Analysis, kostu-unitateak, silexaren hedapena, logika lausoa, Goi Paleolitoa. KEY WORDS: Cost Distance Analysis, Cost Units, dissemination of flint, Fuzzy Logic, Upper Paleolithic.
\end{abstract}

\section{Aitor SÁNCHEZ(1), Eder DOMINGUEZ-BALLESTEROS ${ }^{(2)}$, Maite GARCÍA-ROJAS(3), Alejandro PRIETO(4), Aitor CALVO ${ }^{(5)}$ y Javier ORDOÑO(6)}

\section{RESUMEN}

Los estudios referentes a la gestión de los recursos líticos -especialmente el sílex-por parte de los grupos prehistóricos en las dos vertientes del Pirineo occidental han aumentado de manera considerable en las últimas décadas. En este trabajo, pretendemos realizar una aproximación a la movilidad de los grupos superopaleolíticos, mediante los Sistemas de Información Geográfica (SIG). Estos nos permiten relacionar espacialmente los afloramientos de sílex y los restos líticos encontrados en los yacimientos arqueológicos. Para ello, hemos construido, a través de los Cost Distance Analysis, una serie de mapas que permiten detectar las dinámicas de expansión de cada uno de afloramientos, asi como los patrones de aprovisionamiento de cada yacimiento. Adicionalmente, hemos relacionado los valores numéricos obtenidos del análisis espacial con la cantidad de sílex encontrado en cada nivel arqueológico a partir de estadística descriptiva y la aplicación de la lógica difusa. De esta forma hemos podido revisar los conceptos de autoctonía y aloctonía, así como plantear modelos generales de aprovisionamiento y distribución de sílex en la región.

\section{LABURPENA}

Mendebaldeko Pirinioen bi isurialdeetan historiaurreko taldeek baliabide litikoen -batez ere silexa- inguruan eginiko kudeaketari buruzko azterlanak asko ugaritu dira azken hamarkadetan. Lan honetan, hurbiltze bat egin nahi diogu talde superopaleolitikoen mugikortasunari, Informazio Geografikoko Sistemen (GIS) bidez. Hala, espazioa kontuan hartuta lotu ditzakegu aztarnategi arkeologikoetan aurkitutako silexaren eta hondakin litikoen azaleratzeak. Horretarako, Cost Distance Analysis direlakoen bidez, zenbait mapa eraiki ditugu eta horiei esker, azaleratze bakoitzaren hedatze-dinamikak detektatu ditugu. Aztarnategi bakoitzaren hornitze-ereduak ere eraiki ditugu. Horrez gain, azterketa espazialaren emaitza gisa eskuratu ditugun zenbakizko balioak maila arkeologiko bakoitzean aurkitutako silex kantitatearekin lotu ditugu deskribapenezko estatistikatik abiatuta eta logika lausoa aplikatuta. Hala, autoktonia eta aloktonia kontzeptuak berraztertu ahal izan ditugu eta aipatutako eremuko hornitze-eredu orokorrak eta silexaren banaketa planteatu ditugu.

\footnotetext{
(1) Departamento de Geografía, Prehistoria y Arqueología. Área de Prehistoria. Universidad del País Vasco/Euskal Herriko Unibertsitatea (UPV/EHU). C/Tomás y Valiente s/n 01006 Vitoria-Gasteiz (Álava). aitor.sanchez|@ehu.eus.

ORCID: 0000-0002-6538-3095.

(2) Departamento de Geografía, Prehistoria y Arqueología. Área de Prehistoria. Universidad del País Vasco/Euskal Herriko Unibertsitatea (UPV/ EHU). C/Tomás y Valiente s/n 01006 Vitoria-Gasteiz (Álava). eder.dominguez@ehu.eus ORCID: 0000-0002-7338-8232.

(3) Dipartimento di Studi Umanistici Sezione di Scienze Preistoriche e Antropologiche. Universita di Ferrara. C.so Ercole I d’Este 32. 44100. Ferrara. Italia.

Departamento de Geografía, Prehistoria y Arqueología. Área de Prehistoria. Universidad del País Vasco/Euskal Herriko Unibertsitatea (UPV/ EHU). C/Tomás y Valiente s/n 01006 Vitoria-Gasteiz (Álava). maite.garciar@ehu.eus

(4) Departamento de Geografía, Prehistoria y Arqueología. Área de Prehistoria. Universidad del País Vasco/Euskal Herriko Unibertsitatea (UPV/ EHU). C/Tomás y Valiente s/n 01006 Vitoria-Gasteiz (Álava). alejandro.prieto@ehu.eus ORCID: 0000-0002-3853-525X

(5) Departamento de Geografía, Prehistoria y Arqueología. Área de Prehistoria. Universidad del País Vasco/Euskal Herriko Unibertsitatea (UPV/ EHU). C/Tomás y Valiente s/n 01006 Vitoria-Gasteiz (Álava). aitor.calvo@ehu.eus

ORCID: 0000-0002-3753-8731

${ }^{(6)}$ Departamento de Geografía, Prehistoria y Arqueología. Área de Prehistoria. Universidad del País Vasco/Euskal Herriko Unibertsitatea (UPV/ EHU). C/Tomás y Valiente s/n 01006 Vitoria-Gasteiz (Álava). javier.ordono@ehu.eus
} 


\section{ABSTRACT}

Despite the progress made in recent years, we knew few aspects of the macro- behavior of the Paleolithic societies. Even lower is our information on decisions and conditions that led these groups to settle and/or move along the geography. Fortunately, the recent increment of the interest in paleogeographic analysis, the development of new theoretical models, the dissemination of technical procedures and the progressive incorporation of new computational tools, have made possible the emergence of new approaches to the phenomenon of paleolithic territoriality. In the geographical context of this study, on the southern slope of the Western Pyrenees, the work done by A. Tarriño, have made an essential contribution to this subject. While studies of researchers such as Ch. Normand and F. Bon, among others, have done the same on the northern slope.

The use of Geographic Information Systems (GIS) to study the mobility of human groups, has a long history in the peninsular archaeological research, especially in stages of Recent Prehistory. Nevertheless, in the Paleolithic, this tool has not had a similar development. However, on the Cantabrian coast there are new works that use this tool (using optimal routes) and concepts such as accessibility or cost (among others) in the study of settlement patterns or territorial management of faunistic resources.

In the present study we intend, from the concept "cost", to present an approach to flint collection strategies by Upper Paleolithic groups of the western Pyrenees, with the help of tools such as GIS.

The application of converse tool has been carried out to establish a standardize geographical framework on both sides of the Pyrenees. Using the LCP methodology, we have reflected on a map the directionality and the most favorable to human movement from each archaeological sites, and we have obtained quantitative data $(\mathrm{CU})$ which relate orography and distance. The characterization of this data through the fuzzy logic allow as establishing a criterion to define the autochthonous and allochthonous degree of each variety of flint. Finally, through descriptive statistical approach, we have related this dataset with the percentage of flint from each archaeological layer.

This methodology allows as to approach to the complex mechanisms of recruitment of raw materials. This is based on two overlapping models: one based on the recurring collection and short-range mobility (for obtaining autochthonous flint); and another based on occasional recruitment inserted into a bigger circuit of mobility (reflected by the existence of allochthonous flint). The location of each site marks clear differences between the chosen flint and, in turn, three zones of influence. A western area, with a preponderance of peninsular types; a central area, with a high mobility and flint from both sides of the Pyrenees; and eastern area, with majority presence of northern Pyrenean flint. The factors that create these zones are related to the restrictions set by the terrain. These determine the areas of influence of autochthonous flint: Flysch Kurtzia for the western area, Flysch Bidache and Gaintzurizketa for the central area, and Chalosse and Salies-de-Béarn for the eastern area. Despite the existence of these areas, the presence of certain flint types shows a larger scale mobility, as evidenced by the high distribution of the Trevino, Urbasa and Chalosse flint. The distribution of allochthonous flint and, to a lesser extent, the autochthonous flint, allow us to see small chronological variations and the different functionality of each site.

\section{1.- INTRODUCCIÓN}

A pesar de los avances producidos en los últimos años, pocos son aún los aspectos conocidos de la conducta macroespacial de las sociedades paleolíticas, siendo aún menor nuestra información sobre las decisiones y condicionantes que llevaron a estos grupos a asentarse y/o moverse a lo largo y ancho de la geografía. Por fortuna, una creciente preocupación por los análisis paleogeográficos, el desarrollo de nuevos modelos teóricos y análisis -paleoambientales, de isótopos estables o arqueopetrológicos, entre otros- y la progresiva incorporación de nuevas herramientas computacionales, han hecho posible la aparición de nuevos enfoques en torno al fenómeno de la territorialidad paleolítica (e.g. KELLY, 1995; BINFORD, 2001; BURKE, 2004; GENESTE, et al. 2008).

El incremento de los estudios de materias primas líticas es, desde hace varias décadas la punta de lanza del conocimiento del aprovisionamiento y gestión de los recursos líticos, así como de la articulación de los territorios económicos y los patrones de movilidad de los grupos paleolíticos (TERRADAS, 2002; MANGADO, 2006; DJINDJIAN, 2009). El enorme avance que ha supuesto la localización precisa y la caracterización petrológica de las fuentes de aprovisionamiento de sílex, ha permitido atestiguar, entre otros, la existencia de relaciones efectivas de larga distancia entre hábitats y afloramientos (e.g. DEMARS, 1982; CORCHÓN, et al. 2009). En nuestro marco geográfico, los trabajos desarrollados por A. Tarriño en la vertiente sur del Pirineo Occidental han contribuido de manera esencial a este sujeto (e.g. TARRIÑO, 2006 y 2011a; TARRIÑO, et al. 2007), mientras que los estudios de Ch. Normand y F. Bon, entre otros, han hecho lo propio en la vertiente norte (e.g. BON, et al. 1996; BON, et al. 2002; NORMAND, 2002).

Como resultado, actualmente contamos con un número creciente de "mapas de puntos y flechas", donde quedan expresadas dichas relaciones de forma lineal. En nuestra opinión, éstos no profundizan en los condicionantes geográficos que influyeron en la captación de este recurso, así como en el esfuerzo necesario para abastecerse de materiales que afloran a decenas o centenas de kilómetros de los yacimientos arqueológicos.

Por otro lado, el empleo de los Sistemas de Información Geográfica (de ahora en adelante SIG) en la modelización de la movilidad de los grupos humanos cuenta con una larga trayectoria en la investigación arqueológica peninsular, sobre todo en el Holoceno (e.g. FAIRÉN, 2004; LLOBERA et al. 2011; MURRIETA FLORES, et al. 2011). No obstante, en la investigación del Paleolítico en la Cornisa Cantábrica comienzan a ver la luz trabajos basados en esta herramienta y en conceptos como los de accesibilidad o coste (entre otros), orientados al estudio de los patrones de asentamiento (GARCÍA, 2010; TURRERO, et al. 2013; RÍOS, GARCÍA, 2015; FONTES, 2016) o a la gestión territorial de los recursos faunísticos (COWARD, 2004; MARÍN, 2008).

En el caso del aprovisionamiento de recursos líticos, su aplicación resulta aún si cabe más novedosa, con experiencias piloto como las efectuadas en el valle 
portugués de Foz Côa (AUBRY, et al. 2012) o, ya en la Región Cantábrica, en los valles del Trubia en Asturias (FERNÁNDEZ, 2010) o del Asón en Cantabria (RISSETTO, 2009 y 2012). Estos trabajos pioneros han recogido la preocupación por profundizar en la modelización de la movilidad, permitiendo obtener conclusiones provisionales de interés acerca de los modelos de gestión territorial de los grupos paleolíticos.

En este estudio presentamos una aproximación de los patrones de aprovisionamiento de sílex de los grupos superopaleolíticos del Pirineo occidental a partir del concepto de "Coste", (PRIETO, et al. 2016). Este, basado en los Cost Distance Analysis, permite considerar la orografía de la región gracias a la ayuda de herramientas de análisis cartográfico digital (e.g. VAN LEUSEN, 2002; WHEATLEY, GILLINGS, 2002; CONNOLLY, LAKE, 2006). Además, se analiza el radio de difusión de los distintos afloramientos, así como su expansión. Por último, el "coste" nos permite adquirir una visión basada en la accesibilidad a estos afloramientos, y de esta manera poder establecer, aplicando la lógica difusa, criterios de aloctonía y autoctonía para las múltiples variedades de sílex de la región.

\section{2.- CONTEXTO Y MATERIALES}

El marco geográfico de estudio se circunscribe a las estribaciones occidentales del Pirineo, tanto en su vertiente sur (País Vasco peninsular y Navarra) como norte (País Vasco continental y sur de Landas). Esta área resulta muy apropiada para este análisis (Figura 1). Primero, por ser una zona de paso natural entre la Península lbérica y el resto del continente europeo, en la que se documenta una gran movilidad de los grupos a lo largo del Paleolítico superior (e.g. ARRIZABALAGA, 2007; ARRIZABALAGA, et al. 2013; ARRIZABALAGA, et al. 2014). $Y$, segundo, porque en ambas vertientes del Pirineo occidental existe una importante densidad de afloramientos de sílex de gran calidad ampliamente explotados.

Por ello, hemos seleccionado los afloramientos de sílex más empleados a lo largo del Paleolítico superior en esta región: Flysch Kurtzia (Barrika, Bizkaia), Treviño (Sierra de Araico-Cucho, Burgos), Urbasa (Sierra de Urbasa, Navarra), Flysch Gaintxurizketa (Gaintxurizketa, Gipuzkoa), Flysch Bidache (entre Biarritz y Bidache, Pirineos Atlánticos), Salies-de-Béarn (Peyrehorade, Pirineos Atlánticos) y Chalosse (Audignon-Montaut y Bastennes-Gaujacq, Landas). Estos afloramientos y tipos han sido objeto de estudios geológicos y arqueopetrológicos más o menos pormenorizados, habiendo sido sintetizados en un reciente trabajo (TARRIÑO, et al. 2015).

Por último, han sido objeto de análisis los niveles arqueológicos superopaleolíticos que cuentan con estudios de procedencia de materias primas líticas en los que se ha identificado alguno de los tipos arriba expuestos (Tabla I).

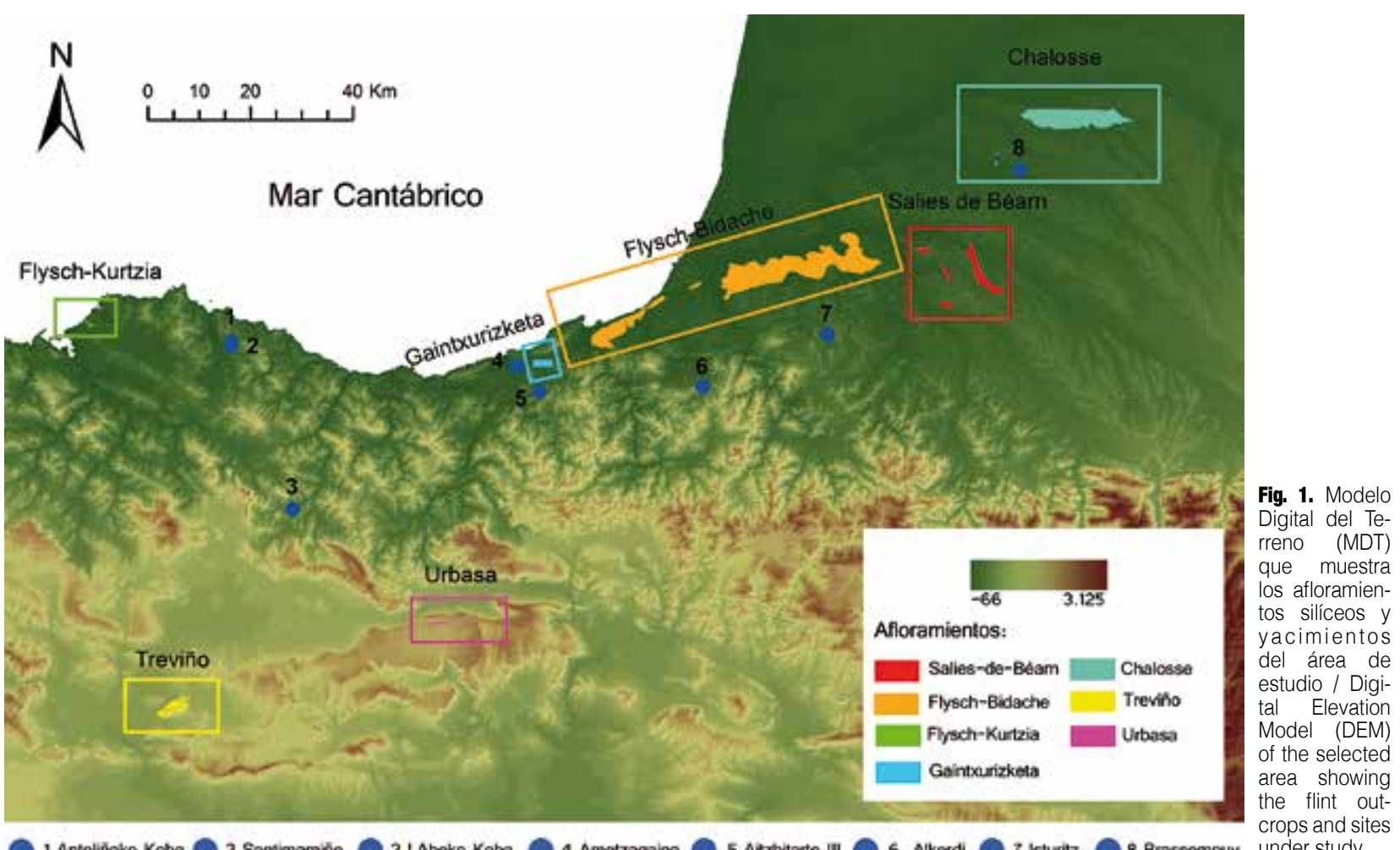

1 Antoliñako Kobe

2 Santimamile

3 Labeko Kobe

4 Ametzagaina

5 Alzbitarte III

6 Akerdi

7 tsturitz

Brassempuy crops and sites 


\begin{tabular}{|c|c|c|c|c|c|c|c|c|c|c|c|c|c|}
\hline \multirow[b]{2}{*}{ Yacimiento } & \multirow[b]{2}{*}{ Localización } & \multirow[b]{2}{*}{ Tipo } & \multirow[b]{2}{*}{ Nivel } & \multirow[b]{2}{*}{ Adscripción } & \multirow{2}{*}{$\begin{array}{c}N^{\circ} \text { restos } \\
\text { analizados }\end{array}$} & \multicolumn{7}{|c|}{ Variedad sílex (\%) } & \multirow[b]{2}{*}{ Bibliografía } \\
\hline & & & & & & $\begin{array}{l}\text { Flysch } \\
\text { Kurtzia }\end{array}$ & Urbasa & Treviño & $\begin{array}{l}\text { Flysch } \\
\text { Gaintx. }\end{array}$ & $\begin{array}{l}\text { Flysch } \\
\text { Bidache }\end{array}$ & $\begin{array}{c}\text { Salies- } \\
\text { de-Béarn }\end{array}$ & Chalosse & \\
\hline \multirow{6}{*}{ Aitzbitarte III } & \multirow{6}{*}{$\begin{array}{c}\text { Rentería } \\
\text { (Gipuzkoa) }\end{array}$} & \multirow{6}{*}{ Cueva } & Vb inf & $\begin{array}{l}\text { Musteriense- } \\
\text { Auriñaciense }\end{array}$ & 46 & 0 & 0 & 0 & 60,9 & 30,4 & 0 & 0 & Tarriño 2011a \\
\hline & & & $\mathrm{Vb}$ & $\begin{array}{l}\text { Auriñaciense } \\
\text { evolucionado }\end{array}$ & 850 & 0 & 6,4 & 0,8 & 42,6 & 28,7 & 0,6 & 2 & Tarriño 2011a \\
\hline & & & Vb sup & Gravetiense & 165 & 0 & 9,1 & 1,8 & 43,6 & 22,4 & 0 & 4,2 & Tarriño 2011a \\
\hline & & & $\mathrm{Va}$ & Gravetiense & 358 & 0 & 10,9 & 0,6 & 19,3 & 39,4 & 4,5 & 11,7 & Tarriño 2011a \\
\hline & & & IV & Gravetiense & 222 & 0 & 19,8 & 0,5 & 16,7 & 43,2 & 0,45 & 8,6 & Tarriño 2011a \\
\hline & & & III & $\begin{array}{l}\text { PS indetermi- } \\
\text { nado }\end{array}$ & 356 & 0 & 16,3 & 0,6 & 10,7 & 53,9 & 0 & 9,55 & Tarriño 2011a \\
\hline \multirow{3}{*}{$\begin{array}{l}\text { Antoliñako } \\
\text { Koba }\end{array}$} & \multirow{3}{*}{$\begin{array}{l}\text { Gauteguiz- } \\
\text { Arteaga } \\
\text { (Bizkaia) }\end{array}$} & \multirow{3}{*}{ Cueva } & $\begin{array}{l}\text { Lab/Lmbk } \\
\text { sup/Lmbk } \\
\text { inf }\end{array}$ & Gravetiense & 224 & 75 & 10,3 & 4,9 & 0 & 0 & 0 & 0 & Tarriño 2006 \\
\hline & & & Lmc & Solutrense & $415^{\star \star * \star * \star}$ & 58,3 & 9,9 & 20,7 & 0 & 0 & 0 & 0 & Tarriño 2006 \\
\hline & & & $\underset{\text { Lanc }}{\text { Lgc/Lamc/ }}$ & $\begin{array}{l}\text { Magdale- } \\
\text { niense }\end{array}$ & 77 & 79,2 & 6,5 & 9,1 & 0 & 0 & 0 & 0 & Tarriño 2006 \\
\hline \multirow{6}{*}{ Labeko Koba } & \multirow{6}{*}{$\begin{array}{l}\text { Arrasate- } \\
\text { Mondragon } \\
\text { (Gipuzkoa) }\end{array}$} & \multirow{6}{*}{ Cueva } & IX & $\begin{array}{l}\text { Chatelperro- } \\
\text { niense }\end{array}$ & 68 & 36,8 & 26,5 & 19,1 & 0 & 0 & 0 & 0 & Tarriño 2000 \\
\hline & & & VII & $\begin{array}{l}\text { Protoauriña- } \\
\text { ciense }\end{array}$ & 1.422 & 7 & 65,5 & 23,3 & 0 & 0 & 0 & 0 & Tarriño 2000 \\
\hline & & & VI & $\begin{array}{l}\text { Auriñaciense } \\
\text { antiguo }\end{array}$ & 95 & 8,5 & 35,1 & 42,6 & 0 & 0 & 0 & 0 & Tarriño 2000 \\
\hline & & & V & $\begin{array}{c}\text { Auriñaciense } \\
\text { antiguo }\end{array}$ & 1.358 & 1,5 & 51,8 & 36,5 & 0 & 0 & 0 & 0 & Tarriño 2000 \\
\hline & & & IV & $\begin{array}{c}\text { Auriñaciense } \\
\text { antiguo }\end{array}$ & 875 & 1,8 & 33,7 & 43 & 0 & 0 & 0 & 0 & Tarriño 2000 \\
\hline & & & III & $\begin{array}{l}\text { PS indetermi- } \\
\text { nado }\end{array}$ & 51 & 90,2 & 3,9 & 5,9 & 0 & 0 & 0 & 0 & Tarriño 2000 \\
\hline \multirow[t]{2}{*}{ Santimamiñe } & \multirow{2}{*}{$\begin{array}{l}\text { Kortezubi } \\
\text { (Bizkaia) }\end{array}$} & \multirow[t]{2}{*}{ Cueva } & $\begin{array}{l}\text { Csn-o/H- } \\
\text { Csn/Camr }\end{array}$ & $\begin{array}{l}\text { Magdalenien- } \\
\text { se inferior }\end{array}$ & 92 & 68,5 & 5,4 & 3,3 & 0 & 0 & 0 & 9,8 & Tarriño 2011b \\
\hline & & & Arcp & Aziliense & 144 & 82,6 & 4,2 & 0 & 0 & 0 & 0 & 2,8 & Tarriño 2011b \\
\hline Alkerdi & $\begin{array}{l}\text { Urdax ( } \mathrm{Na}- \\
\text { varra) }\end{array}$ & Cueva & 2 & Gravetiense & 913 & * & 1,3 & 0 & * & $83,9^{*}$ & 1 & 4,9 & Elorrieta 2010 \\
\hline \multirow[b]{2}{*}{ Ametzagaina } & \multirow{2}{*}{$\begin{array}{l}\text { Donostia-San } \\
\text { Sebastián } \\
\text { (Gipuzkoa) }\end{array}$} & \multirow{2}{*}{$\begin{array}{l}\text { Aire } \\
\text { Libre }\end{array}$} & $\begin{array}{l}\text { Conjunto } \\
\text { Este }\end{array}$ & Gravetiense & 2.029 & 0 & 4 & 0 & 30 & 48 & 0 & 13 & $\begin{array}{l}\text { Arrizabalaga } \\
\text { et al. } 2014\end{array}$ \\
\hline & & & $\begin{array}{l}\text { Conjunto } \\
\text { Oeste }\end{array}$ & $\begin{array}{l}\text { Auriñacien- } \\
\text { se(?)-Grave- } \\
\text { tiense }\end{array}$ & 932 & 0 & 1 & 0 & 54 & 32 & 0 & 5 & $\begin{array}{c}\text { Arrizabalaga } \\
\text { et al. } 2014\end{array}$ \\
\hline \multirow[b]{2}{*}{ Brassempouy } & \multirow{2}{*}{$\begin{array}{l}\text { Brassempouy } \\
\text { (Landas) }\end{array}$} & \multirow[b]{2}{*}{ Cueva } & Chantier I & Gravetiense & $441^{\star \star * *}$ & 0 & 0 & 0 & 0 & 0,2 & 0,2 & $79,6^{* * *}$ & Simonet 2012 \\
\hline & & & $\begin{array}{c}\text { Secteur } \\
\text { GG2 }\end{array}$ & Gravetiense & $94^{* \star \star *}$ & 0 & 0 & 0 & 0 & 3,2 & 0 & $66^{* \star \star}$ & Simonet 2012 \\
\hline \multirow{2}{*}{ Isturitz } & \multirow{2}{*}{$\begin{array}{l}\text { Saint-Martin- } \\
\text { d'Arberoue } \\
\text { (Pirineos } \\
\text { Atlánticos) }\end{array}$} & \multirow{2}{*}{ Cueva } & C4d1j & $\begin{array}{l}\text { Protoauriña- } \\
\text { ciense }\end{array}$ & $98^{\star \star \star \star \star \star *}$ & 0 & 4,1 & 1 & 0 & 68,4 & 5,1 & 11,2 & Elorrieta 2015 \\
\hline & & & C4B1 & $\begin{array}{c}\text { Auriñaciense } \\
\text { antiguo }\end{array}$ & 1.658 & 0 & 0,6 & 1 & 0 & $60,4^{* \star}$ & 6,8 & 14,4 & $\begin{array}{l}\text { Tarriño y Nor- } \\
\text { mand } 2002\end{array}$ \\
\hline
\end{tabular}

Tabla 1: Representación de los distintos yacimientos que han sido objeto de estudio en este trabajo con sus niveles arqueológicos, la cronología de los mismos y la presencia de cada tipo de sílex en los diferentes yacimientos. *Elorrieta incluye en el mismo grupo de "sílex del Flysch" los subtipos de "Bidache", "Gaintxurizketa" y "Kurtzia" (aunque el porcentaje de este último es insignificante). **En el artículo (TARRIÑO Y NORMAND, 2002) se incluyen en el mismo grupo de "sílex del Flysch" los subtipos de "Bidache" e "Iholdy". ${ }^{* * * S i m o n e t ~ d i s c r i m i n a ~ e n t r e ~ l o s ~ d i s t i n t o s ~ s u b t i p o s ~ d e ~ C h a l o s s e . ~ A s i ́, ~ e n ~ e l ~ C h a n t i e r ~ I ~ u n ~ 64,4 \% ~}$ son sílex del diapiro de Bastennes-Gaujacq, un 5,9\% son de la zona de Audignon, y un 9,3\% pueden provenir de cualquiera de los dos afloramientos. En el Secteur GG2, el 9,6\% son del diapiro de Bastennes-Gaujacq, el 50\% de Audignon, y el 6,4\% de cualquiera de las dos. ${ }^{* * \star * E n ~ e l ~ C h a n t i e r ~ I, ~ u ́ n i c a m e n t e ~}$ analiza los núcleos y las armaduras (puntas à cran, laminas y puntas de dorso y de dorso truncadas, etc.). En el Secteur GG2 solo las armaduras. *****Tarriño no especifica las variedades norpirenaicas identificadas, que dice rondan el $0,1 \%$ del total de los restos. ${ }^{* * * * *}$ Nivel con porcentajes provenientes de los artefactos retocados / Summary of the sites under analysis, with reference to the archaeological levels included in the study, their chronology and detailed summary of the flint percentages documented in the archaeological sites. *Elorrieta includes the subtypes "Bidache", "Gaintxurizketa" and "Kurtzia" in the same group of "Flysch's flint" (although the percentage of the latter is insignificant). ${ }^{*}$ In the article (TARRIÑO And Normand, 2002) are included in the group of "Flysch's flint" the subtypes "Bidache" and "Iholdy". "** Simonet discriminate between different subtypes of Chalosse. Thus, in the Chantier I, $64.4 \%$ are flint of the Bastennes-Gaujacq diapir, 5.9\% are from Audignon area, and 9.3\% can come from either outcrops. In Secteur GG2, 9.6\% are from the Bastennes-Gaujacq diapir, 50\% from Audignon, and 6.4\% from either. ${ }^{\star * * *}$ In the Chantier I, only analyzes cores and armors (à cran points, etc.). In Secteur GG2 only the armors. ${ }_{\star \star \star \star \star}$ Tarriño doesn't specify the norther pyrenean varieties, that according to him are around $0.1 \%$ of the remains. ${ }^{\star \star \star \star \star \star}$ Level with percentages of the retouched artifacts. 


\section{3.- METODOLOGÍA}

Para la elaboración de este trabajo nos hemos servido de las herramientas de análisis espacial ofrecidas por el Software ArcGis 10.2 y del Modelo Digital del Terreno MDT25 elaborado por el Instituto Geográfico Nacional (IGN, 2014a) y del Modelo Digital del Terreno MDT75 elaborado por el Institut National de I'Information Géographique et Forestiére (IGN, 2014b). En este caso, nos hemos encontrado ante una incompatibilidad de escala ya que los modelos digitales del terreno ofrecidos por el instituto cartográfico español y francés no concuerdan.

Para solventar este problema hemos optado por adecuar la escala de la cartografía española a la francesa convirtiendo las dimensiones de $25 \times 25 \mathrm{~m}$ por pixel a $75 \times 75 \mathrm{~m}$. Pese a la mayor resolución mostrada por el MDT25 hemos optado por convertirla a un MDT75 con el fin de deformar de la menor forma la realidad. Para ello, hemos construido a partir del MDT25, un mapa de curvas de nivel a escala $75 \mathrm{~m}$ mediante la herramienta Contour tool especificando un intervalo entre curvas de $75 m$ (Figura 2-b). Éste será la base para construir el nuevo ráster con la escala deseada. El siguiente paso ha sido la construcción de una malla de toda la superficie de estudio que contenga datos de altitud; para ello, hemos unido las curvas de nivel mediante una red de triángulos irregulares (TIN). Para este paso hemos utilizado la herramienta Create TIN from freatures tool (Figura 2-c). Finalmente, hemos convertido este mapa a un formato ráster, unificación de $75 \times 75 \mathrm{~m}$ reales (TIN to raster tool) (Figura 2-d). Finalizada esta conversión, hemos realizado un mapa base que abarca todo el área de estudio, georeferenciado según el sistema de coordenadas proyectadas UTM-ETRS 89 (30N).

Para la realización de este trabajo, no hemos tenido en cuenta el relieve submarino, ni agentes como la vegetación, la litología, la red fluvial o los agentes meteorológicos, entre otros. Aunque estos tuvieron una gran influencia en la movilidad de los grupos; sin embargo, conocer con exactitud su afección en cada cronología, impide su inclusión de una forma sistemática (PRIETO et al. 2016).

Posteriormente, y a partir de la unión de los MDT75 del territorio, hemos seguido los mismos pasos que en trabajos previos (PRIETO et al. 2016; GARCÍA-ROJAS, et al. ep) utilizando como medida las Unidades de Coste (de ahora en adelante CU, acrónimo de Cost Units), aunque en este caso, únicamente a partir de cada yacimiento. Para la ubicación de los afloramientos se ha tenido en cuenta las unidades geológicas a las que se adscriben, tal y como están limitadas en los mapas geológicos de la serie MAGNA 1:50.000 proporcionados por el Instituto Geológico y Minero de España (IGME, 2010a, IGME, 2010b, IGME, 2010c). Para los afloramientos ubicados al norte de los Pirineos, se ha tomado como referencia el mapa elaborado por Fernandes et al. (2013) (Figura 3).

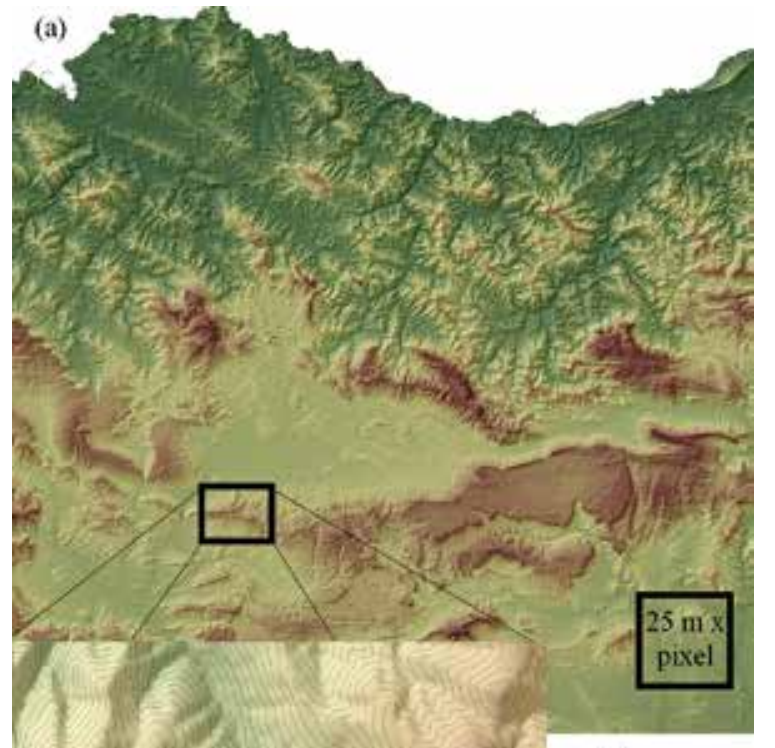

(b)
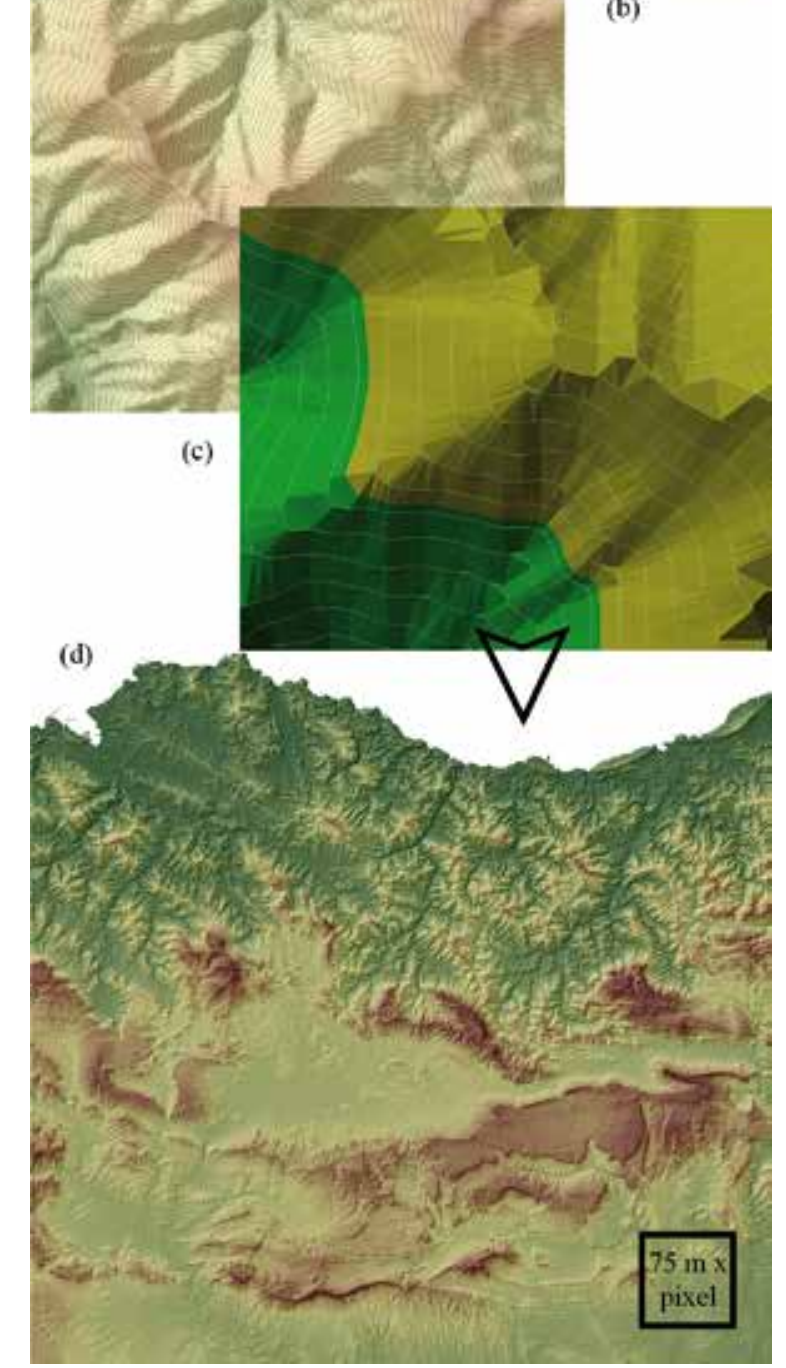

Fig. 2. Proceso de trasformación de escala (de $25 \times 25 \mathrm{~m}$ por pixel a $75 \times 75 \mathrm{~m}$ por pixel) y construcción del nuevo mapa base / DEM scale transformation process (from $25 \times 25 \mathrm{~m} / \mathrm{px}$ to $75 \times 75 \mathrm{~m} / \mathrm{px}$ ) and creation of a new base map. 


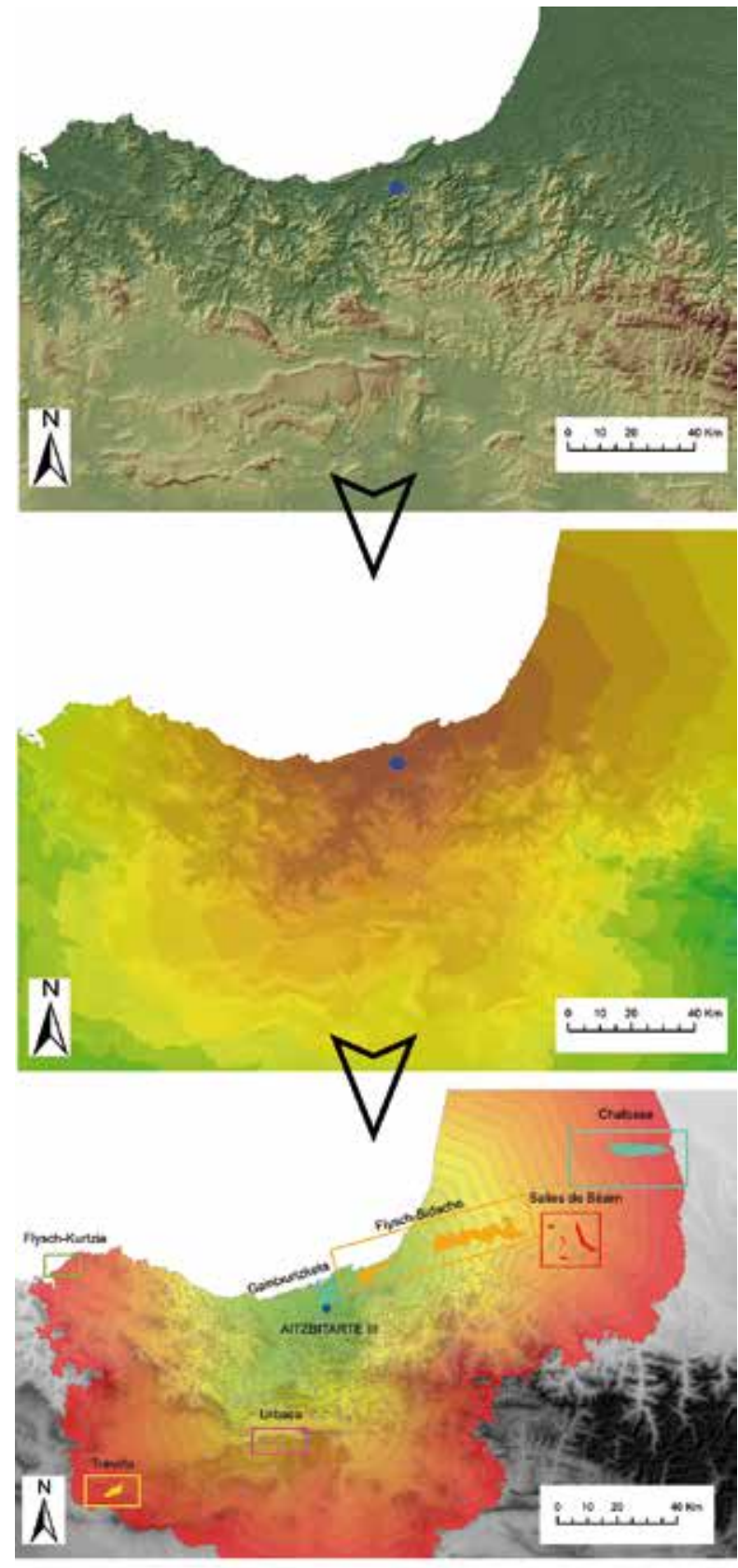

L 5 (4)

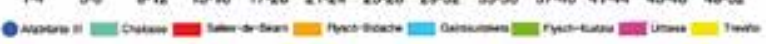

Fig. 3. Construcción del mapa en el que se representan los isocostes que expresan el esfuerzo acumulado que supone desplazarse desde un yacimiento hasta los distintos afloramientos de sílex. Ejemplo de Aitzbitarte III / Example of a cost distance map projected from an archaeological site (Aitzbitarte III), classified by Cost Units (CU), with reference to related flint outcrops.

Además, hemos realizado un análisis estadístico utilizando el Coeficiente de Correlación de Pearson, y $\mathrm{R}^{2}$, con la intención de observar, por un lado, la expansión de los distintos tipos de sílex desde los afloramientos a los yacimientos, y por otro, la relación entre los porcentajes de los diferentes tipos de sílex presentes en cada yacimiento y el esfuerzo que implica la captación de los mismos. Para su mejor visualización hemos representado estos datos en gráficos de dispersión o biplot y en gráficos de cajas y bigotes o boxplot.

Por último, hemos empleado la lógica difusa para analizar los conceptos de autoctonía y aloctonía. Esta disciplina impulsada por el matemático Lotfi A. Zadeh, surge como alternativa a la lógica clásica, que solo permite una clasificación basada en la dualidad verdadero-falso (ZADEH, 1965). La lógica clásica, a la hora de clasificar un elemento dentro de un grupo determinado, solo contempla dos posibilidades: pertenencia al grupo y no pertenencia al grupo. En ocasiones, este sistema no es capaz de representar adecuadamente ciertas situaciones reales. Por ello, la lógica difusa establece la pertenencia a un grupo como algo que puede ser tratado de manera gradual. De esta manera habla de "grado de pertenencia" a un determinado grupo.

\section{4.- RESULTADOS}

En primer lugar, los ocho mapas elaborados ilustran la accesibilidad de cada afloramiento de sílex tomando como referencia la ubicación de los diferentes yacimientos a través de líneas de isocoste. De esta forma podemos conocer la cantidad de esfuerzo, medido en CU necesarias para llegar de un sitio a otro (Tabla II). Además, muestran las unidades geográficas más favorables para el tránsito humano, en la que las líneas de coste muestran un intervalo mayor adecuándose a las morfologías propias del terreno (Anexos). También, hemos detallado la cantidad relativa de cada tipo de sílex en los yacimientos por nivel arqueológico (Tabla I).

La relación entre las CU y los porcentajes de sílex es significativa e inversamente proporcional $(R=-$ 0.592), demostrando una tendencia de a mayor cantidad de esfuerzo, menor cantidad de materia prima. La dispersión de los puntos a través de la gráfica biplot, aun mostrando esta tendencia, refleja una dispersión moderada de los puntos (R2 = 0.355) (Figura 4). Ésta se debe a localización de cada uno de los yacimientos, los condicionantes orográficos de cada uno de los afloramientos, las características composicionales de los tipos de sílex, y la cronología del registro.

Para adentrarnos en estos condicionantes, presentamos un modelo que determina para cada yacimiento arqueológico cuál es el afloramiento que actúa como fuente primaria de captación de sílex, y diferenciarlo de aquellos secundarios o con una influencia reducida. En él, definimos qué materia prima es autóctona o alóctona con respecto a un yacimiento a partir de la lógica difusa (Figura 5). Presentamos un modelo hipotético en el que se categorizan los diferentes tipos de siléx como autóctono $(A)$ o alóctono $(B)$ con relación al yacimiento y en aquellos casos que no se pueden considerar ni uno ni otro son representados por la suma de dos valores (Tabla III). 
Afloramiento

\begin{tabular}{|c|c|c|c|c|c|c|c|}
\hline \multirow{2}{*}{ Yacimiento } & \multicolumn{7}{|c|}{ Afloramiento } \\
\hline & Flysch Kurtzia & Urbasa & Treviño & Flysch Gaintx. & Flysch Bidache & Salies-de-Béarn & Chalosse \\
\hline Aitzbitarte III & 28 & 18 & 27 & 2 & 4 & 20 & 24 \\
\hline Antoliñako Koba & 9 & 24 & 22 & 20 & 23 & 37 & 41 \\
\hline Labeko Koba & 14 & 14 & 12 & 18 & 21 & 35 & 39 \\
\hline Santimamiñe & 9 & 24 & 21 & 21 & 23 & 38 & 42 \\
\hline Alkerdi & 37 & 27 & 37 & 10 & 6 & 15 & 20 \\
\hline Ametzagaina & 27 & 18 & 27 & 1 & 4 & 19 & 24 \\
\hline Brassempouy & 49 & 40 & 50 & 23 & 8 & 5 & 1 \\
\hline Isturitz & 42 & 33 & 42 & 15 & 4 & 7 & 13 \\
\hline
\end{tabular}

Tabla 2: Unidades de Coste (UC) existentes que expresan el esfuerzo que supone acceder a los distintos afloramientos de sílex desde cada yacimiento arqueológico / Cost Units (CU) measured from each site to every flint outcrop, expressing the cost of traveling between them.

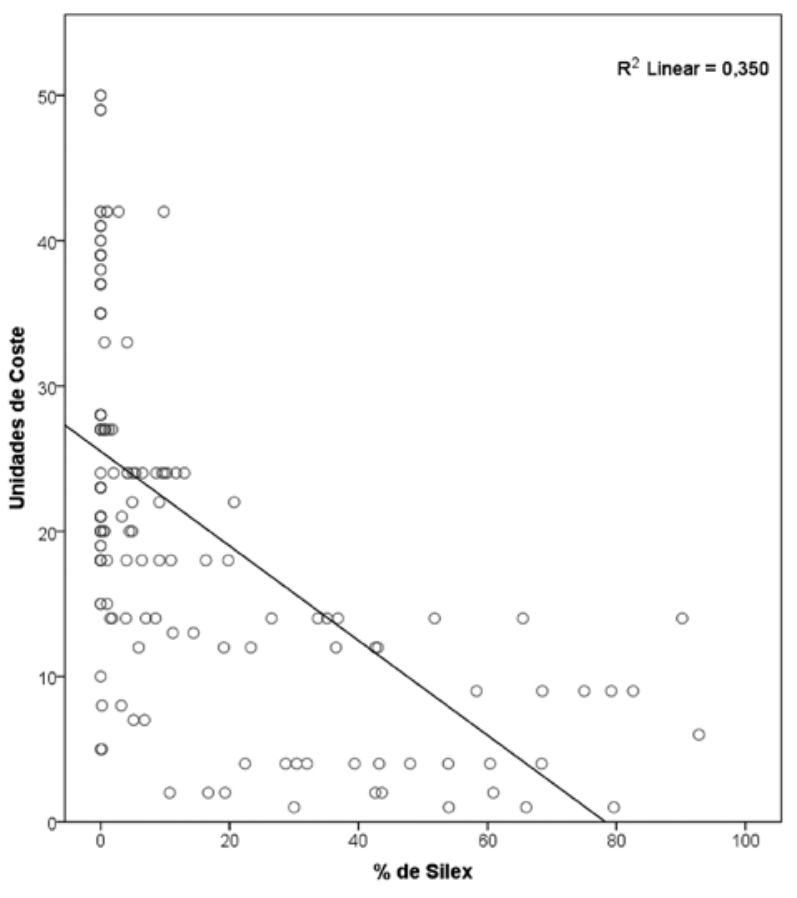

Fig. 4. Relación bivariada entre Unidades de Coste (CU) y porcentaje de sílex trasportado a partir de un gráfico de dispersión / Overall bivariate correlation between flint type percentage and CU.

\section{Autoctonía y aloctonía en función de la accesibilidad}

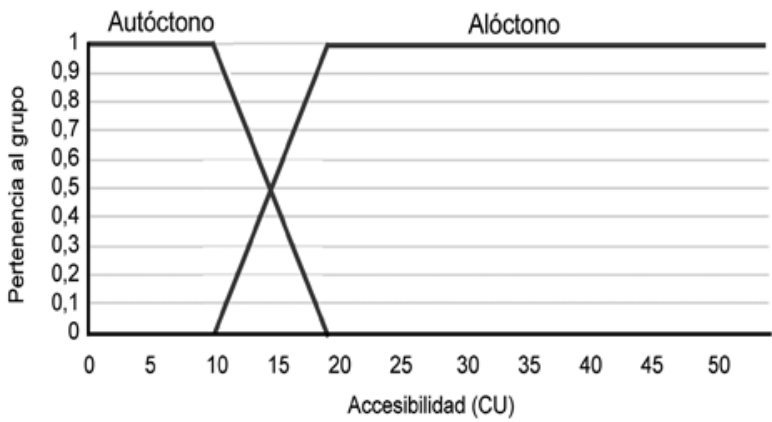

Fig. 5. Grado de pertenencia a cada una de las categorías según la accesibilidad (en CU). Para ciertos valores de costes el grado de pertenencia a un grupo está representado por un único valor, sin embargo, los valores de coste que quedan comprendidos en la llamada "zona difusa" están representados por la suma de dos valores / Degree of membership to each category according to the accessibility (CU). For certain values of cost, the degree of belonging to a group is represented by a single value, however the cost values that fall within the "fuzzy area" are represented by the addition of two values.

\begin{tabular}{|c|c|c|c|c|c|c|c|}
\hline \multirow{2}{*}{ Yacimiento } & \multicolumn{9}{|c|}{ Afloramiento } \\
\cline { 2 - 9 } & Kurtzia & Urbasa & Treviño & Gaintxurizketa & Bidache & Salies-de Bearn & Chalose \\
\hline Aitzbitarte III & $\mathrm{B}^{*}$ & $0,11 \mathrm{~A}+0,88 \mathrm{~B}$ & $\mathrm{~B}$ & $\mathrm{~A}$ & $\mathrm{~A}$ & $\mathrm{~B}$ & $\mathrm{~B}$ \\
\hline Antoliñako Koba & $\mathrm{A}$ & $\mathrm{B}$ & $\mathrm{B}$ & $\mathrm{B}^{*}$ & $\mathrm{~B}^{*}$ & $\mathrm{~B}^{*}$ & $\mathrm{~B}^{*}$ \\
\hline Labeko Koba & $0,55 \mathrm{~A}+0,44 \mathrm{~B}$ & $0,55 \mathrm{~A}+0,44 \mathrm{~B}$ & $\begin{array}{c}0,77 \mathrm{~A} \\
0,22 \mathrm{~B}\end{array}$ & $0,11 \mathrm{~A}+0,88 \mathrm{~B}^{*}$ & $\mathrm{~B}^{*}$ & $\mathrm{~B}^{*}$ & $\mathrm{~B}^{*}$ \\
\hline Santimamiñe & $\mathrm{A}$ & $\mathrm{B}$ & $\mathrm{B}$ & $\mathrm{B}^{*}$ & $\mathrm{~B}^{*}$ & $\mathrm{~B}^{*}$ & $\mathrm{~B}^{*}$ \\
\hline Alkerdi & $\mathrm{B}$ & $\mathrm{B}$ & $\mathrm{B}^{*}$ & $\mathrm{~A}$ & $\mathrm{~A}$ & $0,44 \mathrm{~A}+0,55 \mathrm{~B}$ & $\mathrm{~B}$ \\
\hline Ametzagaina & $\mathrm{B}^{*}$ & $0,11 \mathrm{~A}+0,88 \mathrm{~B}$ & $\mathrm{~B}^{*}$ & $\mathrm{~A}$ & $\mathrm{~A}$ & $\mathrm{~B}^{*}$ & $\mathrm{~B}$ \\
\hline Brassempouy & $\mathrm{B}^{*}$ & $\mathrm{~B}$ & $\mathrm{~B}^{*}$ & $\mathrm{~B}^{*}$ & $\mathrm{~A}$ & $\mathrm{~A}$ & $\mathrm{~A}$ \\
\hline Isturitz & $\mathrm{B}^{*}$ & $\mathrm{~B}$ & $\mathrm{~B}$ & $0,44 \mathrm{~A}+0,55 \mathrm{~B}^{*}$ & $\mathrm{~A}$ & $\mathrm{~A}$ & $0,66 \mathrm{~A}+0,33 \mathrm{~B}$ \\
\hline
\end{tabular}

Tabla 3: Modelo hipotético de definición de un tipo de sílex como autóctono o alóctono con relación al yacimiento en el que aparece (el asterisco indica que un tipo de sílex no está identificado en el yacimiento) / Hypothetical model used to establish whether a flint type is local or alloctonous with regard to the site where it appears (the asterisk indicates that a flint type was not identified at the site). 
Hemos ordenando los niveles cronológicamente, y en ellos, observamos matices en el grado de autoctonía y la aloctonía a lo largo del Paleolítico superior (Figura 6). De esta forma, se aprecia como en los niveles más antiguos, representados principalmente por el yacimiento de Labeko Koba, el grado de aloctonía es más alto. En los niveles temporalmente intermedios se mantiene o aumenta ligeramente la presencia de sílex autóctono, mientras que en los niveles más modernos la presencia del mismo disminuye progresivamente exceptuando el nivel Solutrense de Antoliñako Koba. Por último, En los casos de Aitzbitarte III Vb inf y los dos niveles de Brassempuy, no existe presencia de sílex alóctono.

Teniendo en cuenta estos matices cronológicos, nos hemos adentrado en los otros factores para, por un lado, comprender la dispersión de los distintos tipos de sílex desde los afloramientos y, por otro, acercarnos a los patrones de aprovisionamiento del sílex desde los yacimientos.

Para el primero de los casos hemos representado los datos en dos diagramas de caja y bigote (Figuras 7 y 8), en los que observamos diferentes agrupaciones. Una, formada por Treviño, Urbasa y Chalosse que pre- sentan la máxima dispersión al aparecer en la mayoría de yacimientos y niveles. Estos a su vez presentan pequeños porcentajes, aunque son los únicos que superan el $10 \%$ de sílex en yacimientos situados a más de 20CU. Otra, conformada por Flysch Kurtzia y Flysch Bidache que son los mejor representados de forma global en el conjunto de niveles arqueológicos y presentan una dispersión similar. Posteriormente se encontraría Gaintxurizketa que mantiene porcentajes importantes de materia prima en los niveles en los que aparece y que están situados entre 1 y $2 \mathrm{CU}$, pareciéndose en este aspecto al grupo anterior, pero que presenta la menor dispersión de todos los afloramientos junto con Salies-de-Béarn. Este último con porcentajes muy reducidos incluso en yacimientos como Brassempouy localizado a $5 \mathrm{CU}$.

En el segundo de los casos, hemos agrupado los datos a partir de la gradación suroeste-noreste que se aprecia de los distintos tipos de sílex y que articula el territorio en tres grandes zonas según la influencia de los diferentes afloramientos: una occidental, una central y una oriental.

La occidental está compuesta por los yacimientos de Labeko Koba, Antoliñako Koba y Santimamiñe.

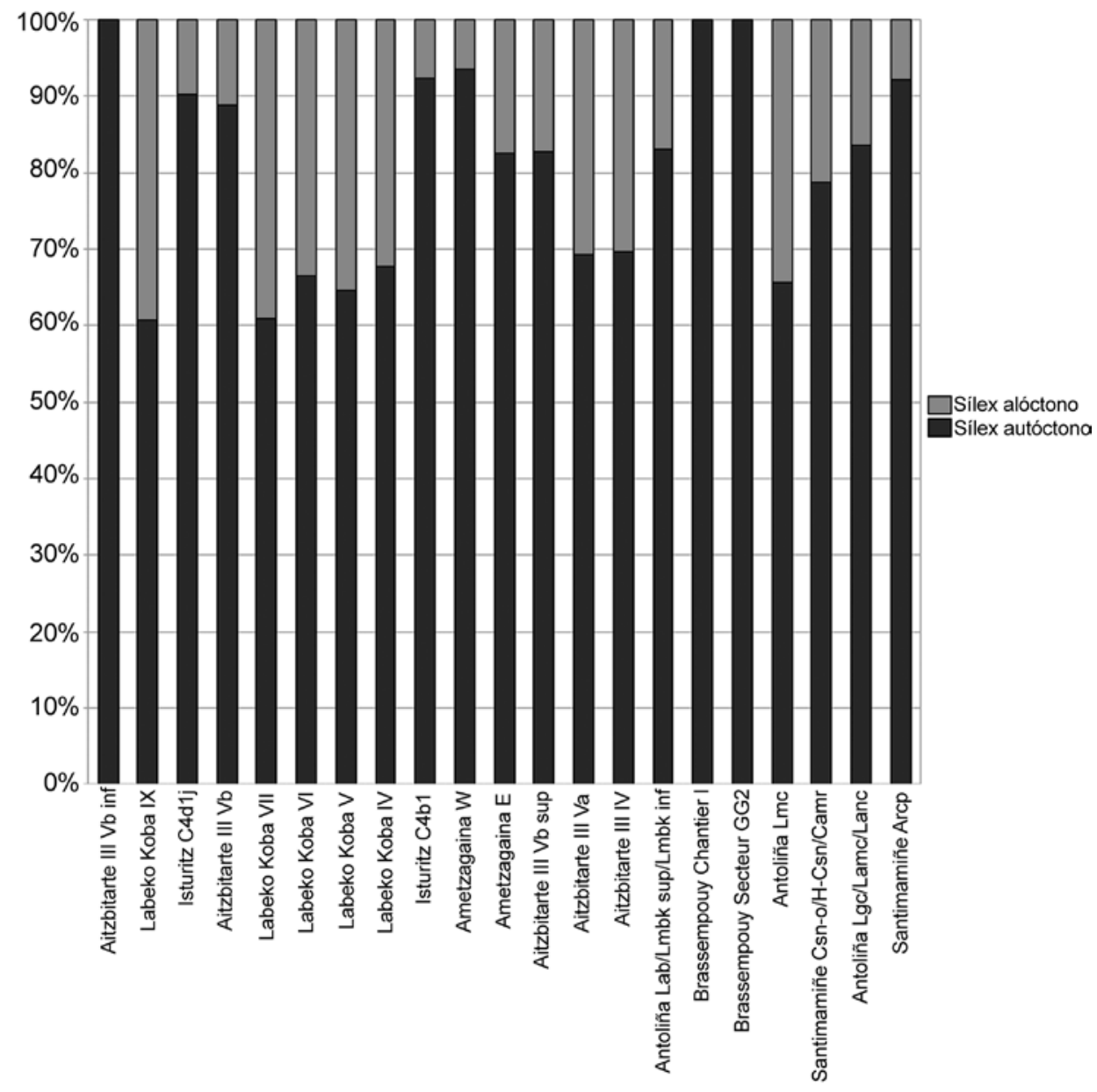

Fig. 6. Ordenación cronológica de todos los niveles arqueológicos con referencia al grado de autoctonía y aloctonía del sílex presente en cada uno de ellos / Chronological arrangement of the archaeological levels under study, with reference to the percentage of local and non-local flint available within them. 

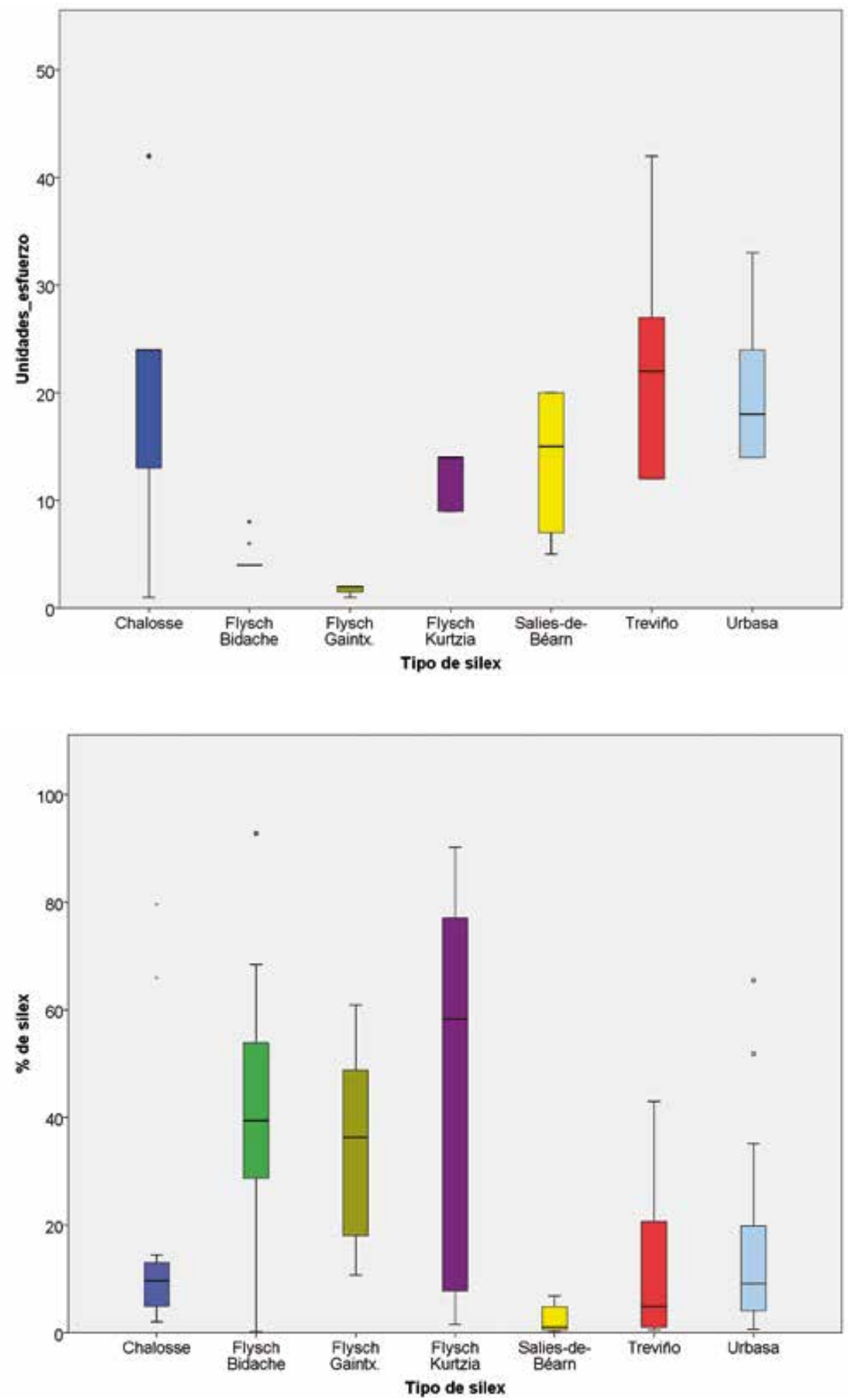

Fig. 7. Diagrama de cajas que muestra la dispersión de cada tipo de sílex mediante la relación entre las unidades de esfuerzo (CU) y la presencia de este en los yacimientos / Boxplot showing the carried flint spread ranges as measured by Cost Units from each outcrop to the archaeological sites, arranged by flint type.
Fig. 8. Diagrama de cajas que muestra la cantidad de sílex transportado a partir de la relación entre el tipo de sílex y su porcentaje global en cada yacimiento / Boxplot that relate the carried flint percentage ranges according to their presence in the archaeological sites, arranged by flint type.
El primero no mantiene la relación inversa entre CU y cantidad de materia prima, además presenta una posición intermedia con respecto a los tres afloramientos de donde provienen sus sílex, todos surpirenaicos y con- siderados parcialmente alóctonos y autóctonos. Además, es el yacimiento en el que se aprecia una mayor variabilidad en los porcentajes de sílex presentes en sus niveles arqueológicos (Tabla III). Antoliñako Koba 
y Santimamiñe, en cambio, mantienen una relación directa entre sus porcentajes de sílex y las CU (Figura 4), representada por Flysch Kurtzia (9CU). También tienen cantidades de sílex autóctonos y alóctonos muy similares, exceptuando el nivel Solutrense de Antoliñako Koba y el Aziliense de Santimamiñe. El primero es el único nivel de esta cronología que presenta mayor cantidad de sílex alóctono (>30\%) (Figura 6). En el segundo, se aprecia un aumento de sílex autóctono y una reducción del alóctono. Sin embargo, observamos algunas diferencias; Antoliñako Koba no tiene sílex norpirenaicos, a diferencia de Santimamiñe, que a partir del Magdaleniense inferior tiene porcentajes de sílex de afloramientos muy poco accesibles como Chalosse (42CU).

La zona central está representada por los yacimientos de Ametzagaina, Aitzbitarte III, Alkerdi e Isturitz. Todos ellos mantienen una relación directa entre sus porcentajes de sílex y las CU (Figura 4), proveyéndose del sílex de Flysch Bidache principalmente. En Ametzagaina y Aitzbitarte III, tanto Gaintxurizketa como Flysch Bidache son los sílex más representados en sus conjuntos y, además, hay una sustitución progresiva a partir del Gravetiense del primero al segundo (Tabla I). Con respecto a los sílex alóctonos, el yacimientos de Aitzbitarte III presenta más tipos de sílex en sus niveles que Ametzagaina, excepto en el Musteriense-Auriñaciense. Por su parte, Alkerdi e Isturitz tienen una presencia mayoritaria de sílex de Flysch Bidache.

La última zona, la oriental, sólo se encuentra Brassempouy que únicamente presenta sílex autóctonos, con una representación mayoritaria de Chalosse (1CU), aunque también tiene porcentajes mínimos de Saliesde-Béarn y de Flysch Bidache.

\section{5.- DISCUSIÓN}

El Paleolítico superior del Pirineo occidental resulta un marco muy apropiado para la utilización de las CU, ya que en él se documenta una reiterada circulación de los sílex tratados en este trabajo entre ambas vertientes, a través de un eje suroeste-noreste representado por el corredor de Txingudi (e.g. TARRIÑO Y NORMAND, 2002; TARRIÑO, 2011a y 2011b; ARRIZABALAGA et al. 2014). Estos flujos entre ambas vertientes del Pirineo también se observan en otros ámbitos de la cultura material (FOUCHER AND SAN JUAN-FOUCHER, 2008).

Antes de analizar los datos, debemos remarcar que existen más variables además del coste, que influyen en los patrones de aprovisionamiento de materias primas de aquellos grupos humanos. Por lo tanto, aunque los factores orográficos suponen una parte importante de los condicionantes físicos que afectan a su movilidad, también debemos considerar otras interpretaciones socio-económicas y culturales (e.g. TERRADAS, 2002; SAUVET et al. 2008; PRIETO, et al. 2016).

\subsection{La dispersión del sílex en el Pirineo occidental}

Teniendo en cuenta los datos ya aportados, hemos diferenciado tres modalidades de difusión de estos sílex.

La primera, la observamos en los casos de Treviño, Urbasa y Chalosse, y se caracteriza por su dispersión amplia y excepcional. Existe una movilidad a gran escala a ambos lados del Pirineo entre 33CU y 42CU a partir de un eje suroeste-noreste y una importante presencia de sílex de Treviño y Urbasa en los yacimientos costeros del País Vasco, mostrando un eje de tránsito y de movilidad recurrente norte-sur desde el interior hacia la costa. Esto puede ser consecuencia de su buena calidad para la talla, así como de la buena accesibilidad que presentan sus afloramientos. Por ello, han sido categorizados como trazadores o, en el caso de Treviño, como super trazador (TARRIÑO et al. 2015). Estos aspectos refuerzan la idea de este espacio geográfico como una vía de comunicación entre la Península Ibérica y el continente Europeo, como muestran también otro tipo de evidencias (ARRIZABALAGA, 2007; ARRIZABALAGA, et al. en este volumen).

La segunda modalidad de dispersión la observamos en el caso del Flysch Kurtzia y Flysch Bidache. Actualmente no nos es posible discriminar en la mayor parte de los casos entre estas dos variedades, lo que conlleva a asociar los restos arqueológicos a una $\mathrm{u}$ otra atendiendo a la proximidad a cada afloramiento. Esto enmascara la presencia de pequeñas cantidades de estos sílex dentro de algunos conjuntos. Aún así, se trata de dos sílex de calidad cuya presencia se ha constatado a distancias considerables (CORCHÓN, et al. 2009), por lo cual han sido categorizados como sílex trazadores (TARRIÑO, et al. 2015). A falta de datos más precisos, podemos interpretarlos como variedades con una dispersión media, pero que nutren también a territorios amplios; así, el sílex de Flysch Kurtzia participa fundamentalmente en los ejes de movilidad norte-sur que comunican la costa con el interior, mientras que Flysch Bidache lo hace en un eje este-oeste hacia ambas vertientes del Pirineo.

La última modalidad se observa en el caso de Gaintxurizketa y Salies-de-Béarn, siendo su dispersión reducida. Esto puede explicarse debido a que sus sílex presentan una calidad menor que el resto y han sido clasificados como sílex regionales (TARRIÑO, et al. 2015).

\subsection{Patrones de movilidad del sílex en el Piri- neo occidental}

La identificación de los distintos tipos de sílex en los niveles arqueológicos nos permite establecer una serie de patrones de movilidad relacionados con la captación de esta materia prima. Los factores principales que influyen en la determinación de dichas pautas están relacionados, en primer lugar, con la ubicación de los yacimientos respecto a los afloramientos y, en segundo lugar, la orografía del terreno. Como resultado, hemos identifica- 
do tres grandes zonas en las que existe una movilidad diferenciada.

La primera se circunscribe al área más occidental del territorio y está representada por los yacimientos de Labeko koba, Antoliñako koba y Santimamiñe. La identificación de los tipos de sílex pone de manifiesto una movilidad noroeste-sur-suroeste, debido a la presencia en todos los niveles de los tres yacimientos de los tipos de Flysch Kurtzia, Urbasa y Treviño.

Esta situación es de carácter general para los yacimientos de Antoliñako koba y Santimamiñe, aunque desde una perspectiva diacrónica se puede apreciar cierta variación. Así durante el Gravertiense y Solutrense en Antoliñako Koba esta dinámica es muy evidente, a partir del Magdaleniense se advierte una diversidad mayor de los tipos de sílex. Echo que se refuerza con la presencia de sílex de Chalosse en Santimamiñe, lo que nos muestra una movilidad de gran recorrido hacia el noreste. En el Aziliense, por su parte, se observa una intensificación de la explotación del sílex autóctono (sílex Flysch Kurtzia) y, en consecuencia, una reducción de la presencia de sílex alóctonos (sílex de Treviño y Urbasa).

El yacimiento de Labeko Koba evidencia una dinámica de captación parcialmente diferente. La presencia de los tres tipos de sílex respalda la movilidad noroeste-sur-suroeste, aunque su ubicación respecto a los tres afloramientos hace que la determinación de cada uno de ellos como autóctono/alóctono varíe a lo largo del tiempo. Así, la elección de un tipo de sílex u otro no responde directamente a una cuestión de accesibilidad, sino a otros aspectos probablemente relacionados con su ubicación estratégica para la gestión del territorio, en la que otros factores socio-ecomómicos tienen mayor peso.

La segunda zona abarca el área central del territorio y está representada por los yacimientos de Ametzagaina, Aitzbitarte III, Alkerdi e Isturitz. La presencia de diferentes sílex en los niveles marca una movilidad variada con una explotación tanto de los afloramientos norpirenaicos como de los surpirenaicos, reflejando una movilidad hacia el noreste, este, sur y sureste. Esta diversidad refleja una alta movilidad en una zona que comunica la Europa continental con la peninsular mediante una orografía excepcional articulada por los corredores naturales de las cuencas de los ríos Deba, Oria o el corredor del Txingudi (Arrizabalaga et al. 2014), que facilitarían el desplazamiento y la comunicación entre estos diferentes espacios.

El tipo de sílex dominante es el de Flysch Bidache, si bien es cierto que la presencia del afloramiento de Gaintxurizketa hace que ambos actúen como autóctonos en los yacimientos de Aitzbitarte III y Ametzagaina. Esta situación se observa en los niveles inferiores de estos yacimientos; durante el Gravetiense, predomina el sílex de Gaintxurizketa, pero éste, posteriormente, se ve sustituido por el de Flysch Bidache, relacionado quizás con una selección de sílex de mejor calidad.
Los tipos de sílex clasificados como alóctonos muestran una mayor diversidad que en la zona anterior, donde cada uno de los yacimientos refleja una movilidad diferente. Así Aitzbitarte III se relaciona con una captación de los sílex de Urbasa y Treviño, dibujando un movimiento hacia el sur-suroeste, aunque en menor medida también al noreste. En Ametzagaina, aparte de presentar menos cantidades y menos tipos de sílex, denota una movilidad más relacionada con los sílex norpirenaicos. Alkerdi e Isturitz indican una movilidad hacia los afloramientos franceses localizados al noreste con una alta presencia de sílex alóctonos (Chalosse y Saliesde-Béarn) en Isturitz, aunque con presencias esporádicas de sílex alóctonos peninsulares.

Por último, la tercera zona es el área más oriental del territorio, representada únicamente por Brassempouy. Este revela patrones de aprovisionamiento que no traspasan los Pirineos. Esto probablemente sea debido a la situación geográfica del yacimiento, localizado junto a uno de los sílex de mayor calidad y dispersión del territorio como es Chalosse. De esta forma, es posible que Brassempouy esté relacionado con la agregación de grupos que presentan una movilidad más circunscrita al área norpirenaica.

\section{CONCLUSIONES}

El presente trabajo constituye una aproximación hacia los procesos de aprovisionamiento de sílex en las estribaciones pirenáicas occidentales. La aplicación de herramientas de conversión de datos espaciales basados en los SIG, nos ha permitido construir un mapa topográfico estandarizado entre ambas partes de los Pirineos. A partir de los Cost Distance Analyse, hemos cartografiado la direccionalidad y las áreas más favorables para el tránsito desde cada yacimiento, y hemos obtenido datos cuantitativos (CU) con los que relacionar las variables orografía y distancia. La caracterización de estos datos a partir de la lógica difusa nos ha permitido establecer criterios para definir en cada yacimiento relaciones de autoctonía y aloctonía para cada sílex. Finalmente, mediante la estadística descriptiva, hemos relacionado estos datos junto con la cantidad de sílex existente en cada nivel arqueológico.

Todo esto, nos ha permitido acercarnos a complejos mecanismos de captación de materia prima basados en dos modelos superpuestos en la mayoría de los yacimientos: uno basado en la captación recurrente y circuitos de movilidad de corto alcance para la obtención de sílex autóctonos; y otro, basado en la captación esporádica inserta en circuitos de movilidad amplia, reflejada por la existencia de sílex alóctonos. La situación geográfica de cada yacimiento marca claras diferencias entre los sílex seleccionados y, a su vez, tres zonas de influencia. Una zona occidental, con una presencia mayoritaria de tipos peninsulares; una central, de alta movilidad y variabilidad de sílex de ambas vertientes de los Pirineos; y una zona oriental, con presencia ma- 
yoritaria de sílex norpirenaicos. Los factores que generan estas zonaciones hay que buscarlos en las limitaciones marcadas por la orografía, pues determinan las áreas de influencia de los sílex autóctonos: Flysch Kurtzia para los primeros, Flysch Bidache y Gaintzurizketa para los segundos y Chalosse y Salies-de-Béarn para los últimos. Pese a la existencia de estas zonas, la evidencia de ciertos tipos silíceos hace ver una movilidad de mayor escala, como evidencia la alta distribución de los sílex de Treviño, Urbasa y Chalosse, aunque con mayor profusión en zonas más accesibles. La variabilidad en la distribución de los sílex alóctonos y en menor medida de los autóctonos, permite observar pequeñas variaciones cronológicas.

Para concluir, esta primera aproximación a la comprensión de los patrones de captación de sílex aplicando los SIG, nos ha permitido abrir una nueva línea de investigación. Esta se verá reforzada por la incorporación de otras perspectivas de análisis como la profundización en las características petrológicas y morfo-métricas de cada tipo de sílex. Así como en la aplicación de caracteres descriptivos del material lítico a partir de la Tipología, Tecnología y/o Traceología. Finalmente, la introducción de datos provenientes del estudio de otros materiales, como el arte mueble o rupestre, o relativos a la captación de recursos biológicos, aportará información crucial para entender la movilidad de estos grupos y sus comportamientos culturales, sociales y económicos.

\section{AGRADECIMIENTOS}

Este trabajo ha sido posible gracias a la financiación parcial del Grupo de Investigación en Prehistoria del Gobierno Vasco (UPV/EHU IT622-13), a la red de trabajo Palmesopyr (CTP2012-R1) y por los proyectos de investigación del Ministerio de Economía y Competitividad de España (HAR2014-53536-P y HAR2015-67429-P). Además, A.S., A.P. y A.C. están financiados por el programa Predoctoral del Departamento de Educación, Lingüística y Cultura del Gobierno Vasco (BFI-2012-205, BFI-2012-121 y BFI-2011-101) y M.G.R por el programa Postdoctoral del Departamento de Educación, Lingüística y Cultura del Gobierno Vasco (POS-2015-1-0053).

\section{BIBLIOGRAFÍA}

\section{ARRIZABALAGA, A.}

2007 Frontières naturelles, administratives et épistémologiques: L'unité d'analyse dans l'archéologie du Paléolithique (dans le cas basque), en CAZALS, N., GONZÁLEZ, J. \& TERRADAS, X. (eds) Frontières naturelles et frontières culturelles dans les Pyrénées préhistoriques, 27-37. PubliCan-Ediciones de la Universidad de Cantabria. Santander.

ARRIZABALAGA, A., CALVO, A., ELORRIETA, I., TAPIA, J. \& TARRIÑO, A.

2014 Where to and what for? Mobility patterns and lithic resources management among Gravettian hunter-gatherers in the Western Pyrenees. Journal of Anthropological Research 70, 233-261.
ARRIZABALAGA, A., IRIARTE-CHIAPUSSO, M. J. \& ORDOÑO, J.

2013 Rivers, Mountains and Footpaths: the Basque Crossroads in the Framework of Palaeolithic Settlement in the Iberian Peninsula, en PASTOORS, A. \& AUFFERMANN B. (eds) Pleistocene foragers on the Iberian Peninsula: their culture and environment. Festschrift in honour of Gerd-Christian Weniger for his Sixtieth Birthday, 25-34. Wissenschaftliche Schriften des Neanderthal Museum. Mettmann.

ARRIZABALAGA, A., PRIETO, A., DOMINGUEZ-BALLESTEROS, E., GARCIA-IBAIBARRIAGA, N., OCHOA, B., ORDONOO, J., ROMERO, A., VILLAUELGA, A., TAPIA, J., AYERDI, M., ECHAZARRETA, A. HERNANDEZ-BELOQUI, B., MEDINA, M.A., BRADTMOLLËR, M., SUAREZ, A., SARASKETA, I. \& IRIARTE-CHIAPUSSO, M.J.

(en este volumen) En la ruta occidental del poblamiento de Iberia. Munibe Monográficos.

AUBRY, T., LUÍS, L., MANGADO, J. \& MATIAS, H.

2012 We will be known by the tracks we leave behind: Exotic lithic raw materials, mobility and social networking among the Côa Valley foragers (Portugal). Journal of Anthropological Science 31, 528-550.

BINFORD, L. R.

2001 Constructing Frames of Reference. An Analytical Method for Archaeological Theory Building Using Huntergatherer and Environmental Data Sets. University of California Press. Berkeley.

BON, F., CHAUVAUD, D., DARTIGUEPEYROU, S., GARDÈRE, PH. \& MENSAN, R

1996 La caractérisation du silex de Chalosse. Antiquités nationales 28, 33-38.

BON, F., CHAUVAUD, D., DARTIGUEPEYROU, S., GARDÈRE, PH, KLARIC, L. \& MENSAN, R.

2002 Les ressources en silex de la Chalosse centrale: gîtes et ateliers du dòme diapir de Bastennes-Gaujacq et de l'anticlinal d'Audignon, en CAZALS, N. (ed), Comportements techniques et économiques des sociétés du Paléolithique supérieur dans le contexte pyrénéen, Projet Collectif de Recherche Service Regional de l'Archéologie de Midi-Pyrénées, 47-63.

BURKE, A.

2004 Alternate Models of Spatial Organisation of Pleistocene Hunter-Gatherers, en CROTHERS G. M. (ed) Hunters and Gatherers in Theory and Archaeology. 192-210. Center for Archaeological Investigations, Southern IIIinois University. Carbondale.

CONNOLLY, J. \& LAKE, M. (eds)

2006 Geographical Information Systems in Archaeology. Cambridge: Cambridge University Press.

CORCHÓN, M. S., MARTÍNEZ, J. \& TARRIÑO, A.

2009 Mobilité, territoires et relations culturelles au début du Magdalénien moyen cantabrique: nouvelles perspectives, en DJINDJIAN, F., KOZLOWSKI, J. \& BICHO, N. (eds) Le concept de territoires dans le Paléolithique supérieur européen. Actes du XVe Congrès mondial (Lisbonne, 4-9 septembre 2006), 217-230. BAR International Series. Oxford. 
COWARD, F.

2004 Transitions, Change and Identity: the Middle and Upper Palaeolithic of Vasco-Cantabrian Spain. University of Southampton, Southampton.

DEMARS, P. Y.

1982 L'utilisation du silex au Paléolithique supérieur: choix, approvisionnement, circulation. L'exemple du bassin de Brive (Vol. 5). CNRS. París

DJINDJIAN, F.

2009 Le concept de territoires pour les chasseurs cueilleurs du Paléolithique supérieur européen, en DJINDJIAN, F. KOZLOWSKI, J \& BICHO, N. (eds) Le concept de territoires dans le Paléolithique supérieur européen. Actes du XVe Congrès mondial (Lisbonne, 4-9 septembre 2006) 3-25. BAR International Series. Oxford.

\section{ELORRIETA, I.}

2010 El aprovechamiento y disponibilidad de las materias primas silíceas durante el Paleolítico Superior en el Pirineo occidental: El caso de Alkerdi como paradigma, Trabajo para la obtención del Diploma de Estudios Avanzados, Universidad del País Vasco-Euskal/Herriko Unibertsitatea (UPV-EHU), Inédito.

2015 Los artefactos retocados del nivel C4d1j de Isturitz: Estudio arqueopetrológico desde la Tipología Analítica, en CALVO, A., SÁNCHEZ, A., GARCÍA-ROJAS, M. \& ALONSO-EGUILUZ, M (eds), Seis décadas de Tipología Analítica. Actas en homenaje a Georges Laplace, 156-166. Vitoria-Gasteiz.

FAIRÉN, S.

2004 ¿Se hace camino al andar? Influencia de las variables medioambientales y culturales en el cálculo de caminos óptimos mediante SIG. Trabajos de Prehistoria 61(2), 25-40.

FERNANDES, P., RAYNAL, J. P., TALLET, P., TUFFERY, C., PIBOULE, M., SÉRONIE-VIVIEN, M., SÉRONIE-VIVIEN, M. R. TURQ, A., MORALA A., AFFOLTER, J., MILLET, D., MILLET, F., BAZILE, F., SCHMIDT, P., FOUCHER, P., DELVIGNE, V., LIAGRE, J., GAILLLOT, S., MORIN, A., MONCEL, M. H., GARNIER, J. F. \& LÉANDRI-BRESSY, C.

2013 Une carte et une base de données pour les formations à silex du sud de la France: un outil pour la pétroarchéologie. PALEO 24, 219-228.

\section{FERNÁNDEZ, J}

2010 Una aportación desde la arqueología del paisaje al conocimiento del primer poblamiento humano del Valle del Trubia. Estudio geoarqueológico y análisis SIG del territorio. Ediciones de la Universidad de Oviedo. Oviedo.

FONTES, L. M.

2016 The Initial Magdalenian mosaic: New evidence from Urtiaga cave, Guipúzcoa, Spain. Journal of Anthropological Archaeology 41, 109-131.

\section{FOUCHER, P. \& SAN JUAN-FOUCHER, C.}

2008 Du silex, de l'os et des coquillages: matières et espaces géographiques dans le Gravettien pyrénéen, en AUBRY, T., ALMEIDA, F., ARAÚJO, A. C. \& TIFFAGOM M. (eds) Space and Time: Which Diachronies, Which Synchronies, Which Scales? Typology vs. Technology.
Actes du XVe Congrès Mondial de I'Union Internationale des Sciences Préhistoriques et Protohistoriques (UISPP) de Lisbonne. BAR International Series 1831 45-55. BAR International Series 1831. Oxford, England.

GARCÍA, A

2010 Patrones de asentamiento y ocupación del territorio en el Cantábrico Oriental al final del Pleistoceno. Una aproximación mediante SIG. Universidad de Cantabria, Santander.

GARCÍA-ROJAS, M., PRIETO, A., SÁNCHEZ, A., CAMARERO, C. \& ZAPATA L.

(Ep) Application of GIS to flint management studies during the Pleistocene to the Holocene transition: the case of Baltzola (Dima, Bizkaia, Spain). Sidestone Press

GENESTE, J. M., CASTEL, J. C. \& CHADELLE, J. P.

2008 From physical to social landscapes: multidimensional approaches to the archaeology of social place in the european Upper palaeolithic, en DAVID, B. \& THOMAS, J. (eds) Handbook of Landscape Archaeology. 228236. Left Coast Press Inc. Walnut Creek, California.

IGME.

2010a Algorta 37-4, MAGNA 2a Ed., Instituto Geológico y Minero de España, Madrid.

2010b. Altsasu 114-3, MAGNA 2a Ed., Institito Geológico y Minero, Madrid.

2010c. Treviño 138-3, MAGNA $2^{a}$ Ed., Instituto Geográfico Minero, Madrid.

IGN.

2014a. Centro de descargas, Ministerio de Fomento, Gobierno de España, http://centrodedescargas.cnig.es/CentroDescargas/index.jsp.

2014b. Institut National de I'Information Géographique et Forestiére, République Française, http://www.geoportail. gouv.fr/donnee/51/cartes-ign.

KELLY, R. L.

1995 The foraging spectrum. Diversity in hunter-gatherer lifeways. Percheron Press. New York

LLOBERA, M., FÁBREGA-ÁLVAREZ, P. \& PARCERO-OUBIÑA, C.

2011 Order in Movement a GIS approach to accessibility. Journal of Archaeological Science 38, 843-851.

MANGADO, J.

2006 El aprovechamiento en las materias primas líticas: Hacia una caracterización paleocultural de los comportamientos paleoeconómicos. Trabajos de Prehistoria 63 (2), 79-91

MARÍN, A. B.

2008 Patrones de movilidad y control del territorio en el Cantábrico Oriental durante el Tardiglaciar. Trabajos de Prehistoria 65, 29-45.

MURRIETA FLORES, P. A., WHEATLEY, D. \& GARCÍA SANJUÁN, L.

2011 Movilidad y vías de paso en los paisajes prehistóricos: megalitos y vías pecuarias en Almadén de la plata, en MAYORAL HERRERA. V \& Celestino Pérez, S. (eds) 
Tecnologías de información geográfica y análisis arqueológico del territorio. Actas del V Simposio Internacional de Arqueología de Mérida, 1-16. Instituto de Arqueología-Mérida. CSIC-Junta de Extremadura-Consorcio de Mérda. Mérida.

\section{NORMAND, C.}

2002 Les ressources en matiéres premiéres siliceuses dans la basse vallée de l'Adour et de ses affluents, en CAZALS, N. (ed.) Comportements techniques et économiques des sociétés du Paléolithique supérieur dans le contexte pyrénéen. Project Collectif de Recherche 2002. Service Regional de l'Archéologie de Midi-Pyrénées, 26-46.

PRIETO, A., GARCÍA-ROJAS, M., SÁNCHEZ, A., CALVO, A., DOMÍNGUEZ-BALLESTEROS, E., ORDOÑO, J. \& GARCÍA-COLLADO, M.

2016 Stones in Motion. Cost units for understanding flint procurement strategies through GIS during the Upper Palaeolithic in the south-western Pyrenees. Journal of Lithic Studies 3 (1), ep.

RÍOS, J. \& GARCÍA, A.

2015 Middle Paleolithic Mobility Patterns and Settlement System Variability in the Eastern Cantabrian Region (Iberian Peninsula): A GIS-Based Resource Patching Model, en CONARD, N. \& DELANGES, A. (eds.). Settlement Dynamics of the Middle Palaeolithic and Middle Stone Age. 329-360. Tübingen: Publications in Prehistory.

\section{RISSETTO, J. D.}

2009 Late Pleistocene Hunter-Gatherer mobility patterns and lithic exploitation in Eastern Cantabria (Spain). University of New Mexico, Albuquerque.

2012 Using Least Cost Path Analysis to Reinterpret Late Upper Paleolithic Hunter-Gatherer Procurement Zones in Northern Spain, en WHITE, D. A. \& SURFACE-EVANS S. L. (eds) Least Cost Analysys of Social Landscapes. Archaeological Case Studies, 11-31. University of Utah Press. Salt Lake City.

SAUVET, G., FORTEA, J., FRITZ, C. \& TOSELLO, G.

2008 Echanges culturels entre groupes humains paléolithiques entre 20.000 et 12.000 BP. Bulletin de la Société Préhistorique Ariège-Pyrénées 63, 73-92.

TARRIÑO, A.

2000 Estudio de la procedencia de los sílex recuperados en el yacimiento de Labeko Koba (Arrasate, País Vasco), en ARRIZABALAGA, A. \& ALTUNA, J. (eds) Labeko Koba (País Vasco): Hienas y Humanos en los albores del Paleolítico superior, 345-354. Munibe.

2006 El sílex en la cuenca vasco-cantábrica y Pirineo navarro: caracterización y su aprovechamiento en la Prehistoria (Vol. 21). Ministerio de Educación y Cultura. Madrid.

2011a Procedencia de los sílex de la cueva de Aitzbitarte-III (Rentería, Gipuzkoa), en ALTUNA,J., MARIEZKURRENA, K. \& RÍOS, J. (eds) Ocupaciones humanas en Aitzbitarte III (País Vasco) 33.600-18.400 BP.: 353-373. EKOB Euskal Kultura Ondare Bilduma. Vitoria-Gasteiz.

2011b Procedencia de los sílex de la industria lítica del yacimiento en cueva Santimamiñe (Kortezubi, Bizkaia), en LÓPEZ QUINTANA, J. C. (coord) La cueva de Santimamiñe, revisión y actualización (2004-2006), 281-290. Diputación Foral de Bizkaia, Bilbao.
TARRIÑO, A. \& NORMAND, C.

2002 Procedencia de los restos líticos en el Auriñaciense Antiguo (C 4b1) de Isturitz (Pyrénées-Atlantiques, Francia), en MAílLO, J.M. (ed) Autour des concepts de Protoaurignacien, d'Aurignacien initial et ancien. Espacio Tiempo y Forma, Serie I, Prehistoria y Arqueología $15,135-143$.

TARRIÑO, A.. OLIVARES, M., ETXEBARRIA, N., BACETA, J. I., LARRASOAÑA, J., YUSTA, I., PIZARRO, J., CAVA, A., BARANDIARÁN, I. \& MURELAGA, X.

2007 El sílex de tipo "Urbasa": Caracterización petrológica y geoquímica de un marcador litológico en yacimientos arqueológicos del Suroeste europeo durante el Pleistoceno superior y Holoceno inicial. Geogaceta 43, 127-130.

TARRIÑO, A., ELORRIETA, I. \& GARCÍA-ROJAS, M.

2015 Flint as raw material in prehistoric times: Cantabrian Mountain and Western Pyrenees data, Quaternary International 364, 94-108.

\section{TERRADAS, $X$.}

2002 La gestión de los recursos minerales en las sociedades cazadoras-recolectoras. Treballs D'Etnoarqueologia, 4.

TURRERO, P., DOMÍNGUEZ-CUESTA, M.J., JIMÉNEZ-SÁNCHEZ, M. \& GARCÍA-VÁZQUEZ, E.

2013 The spatial distribution of Palaeolithic human settlements and its influence on palaeoecological studies: a case from Northern Iberia, Journal of Archaeological Science 40, 4127-4138

\section{VAN LEUSEN, P. M.}

2002 Pattern to Process: Methodological Investigations into the Formation and Interpretation of Spatial Patterns in Archaeological Landscapes. University of Groningen, Groningen.

WHEATLEY, D. \& GILLINGS, M.

2002 Spatial Technology and Archaeology: The Archaeological Applications of GIS. Taylor \& Francis. New York

ZADEH L. A.

1965 Fuzzy Sets. Information and Control 8, 338-353. 

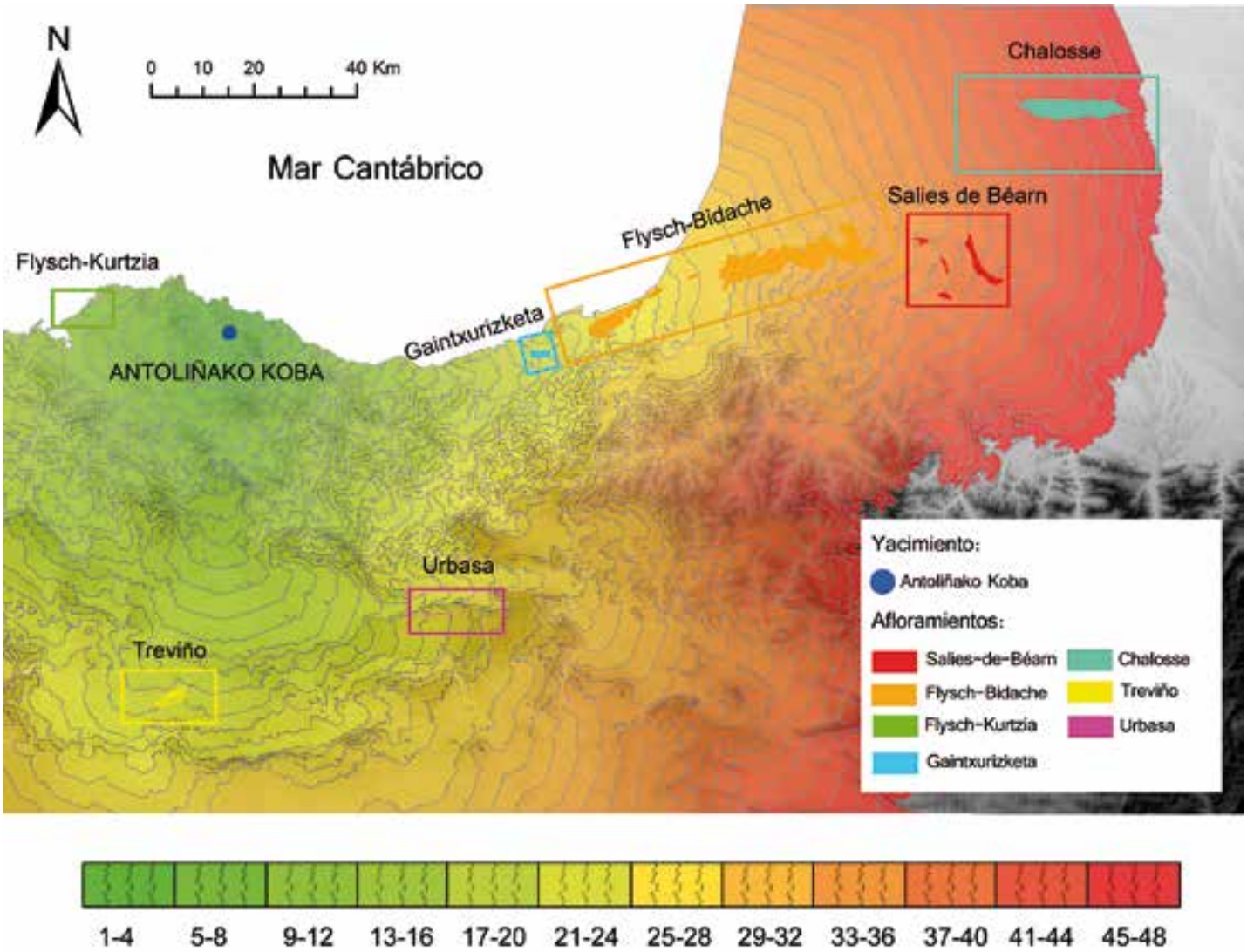
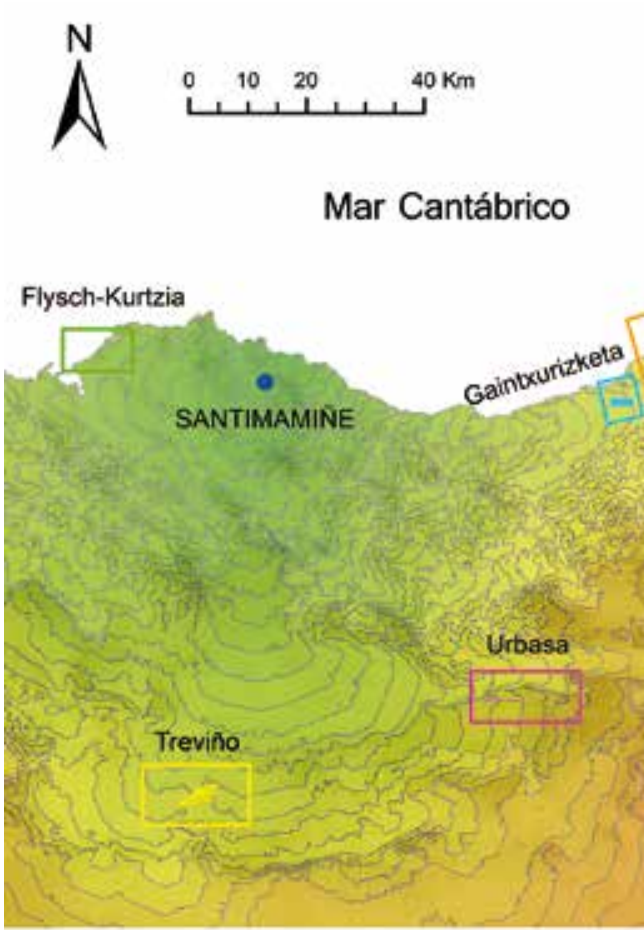
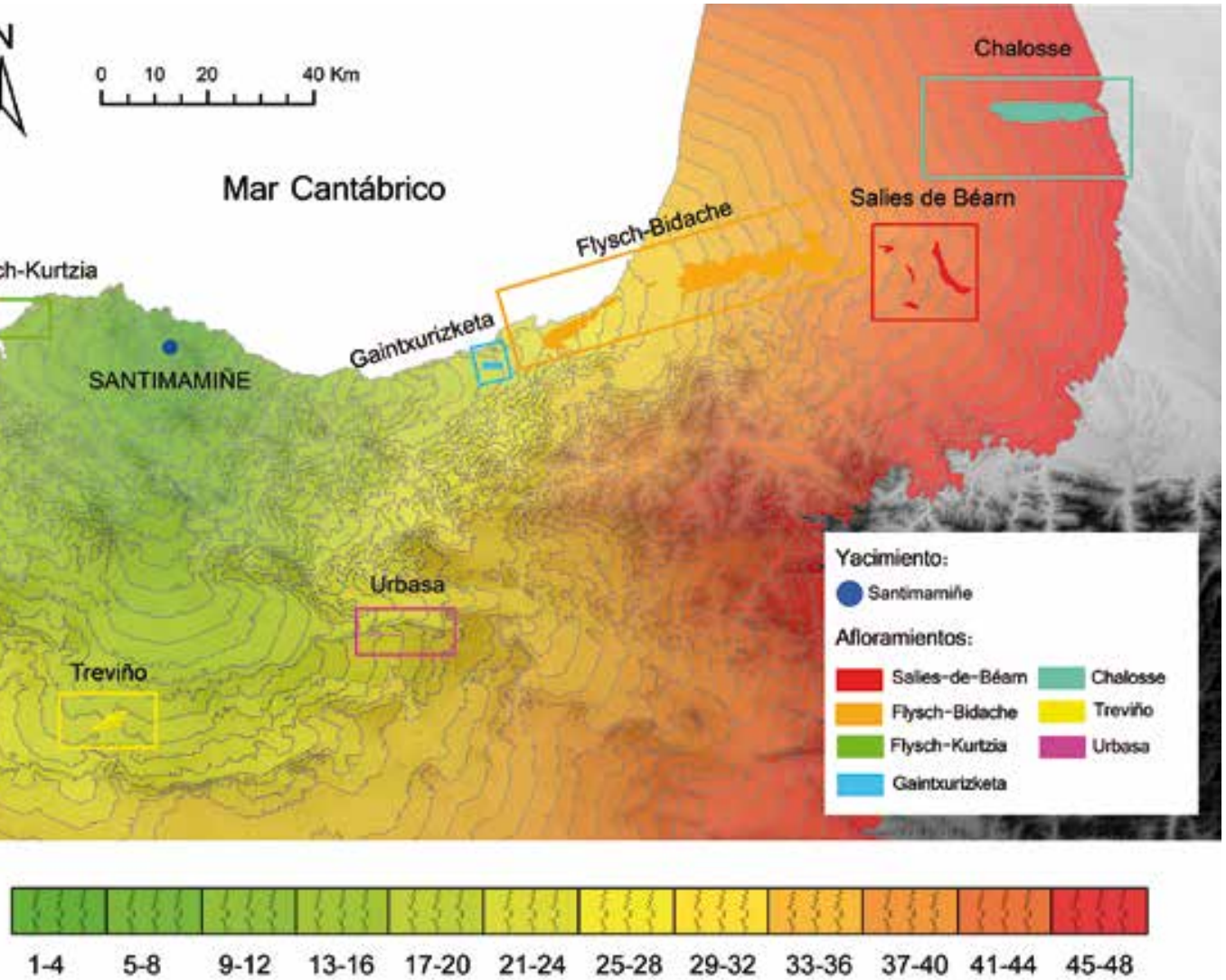

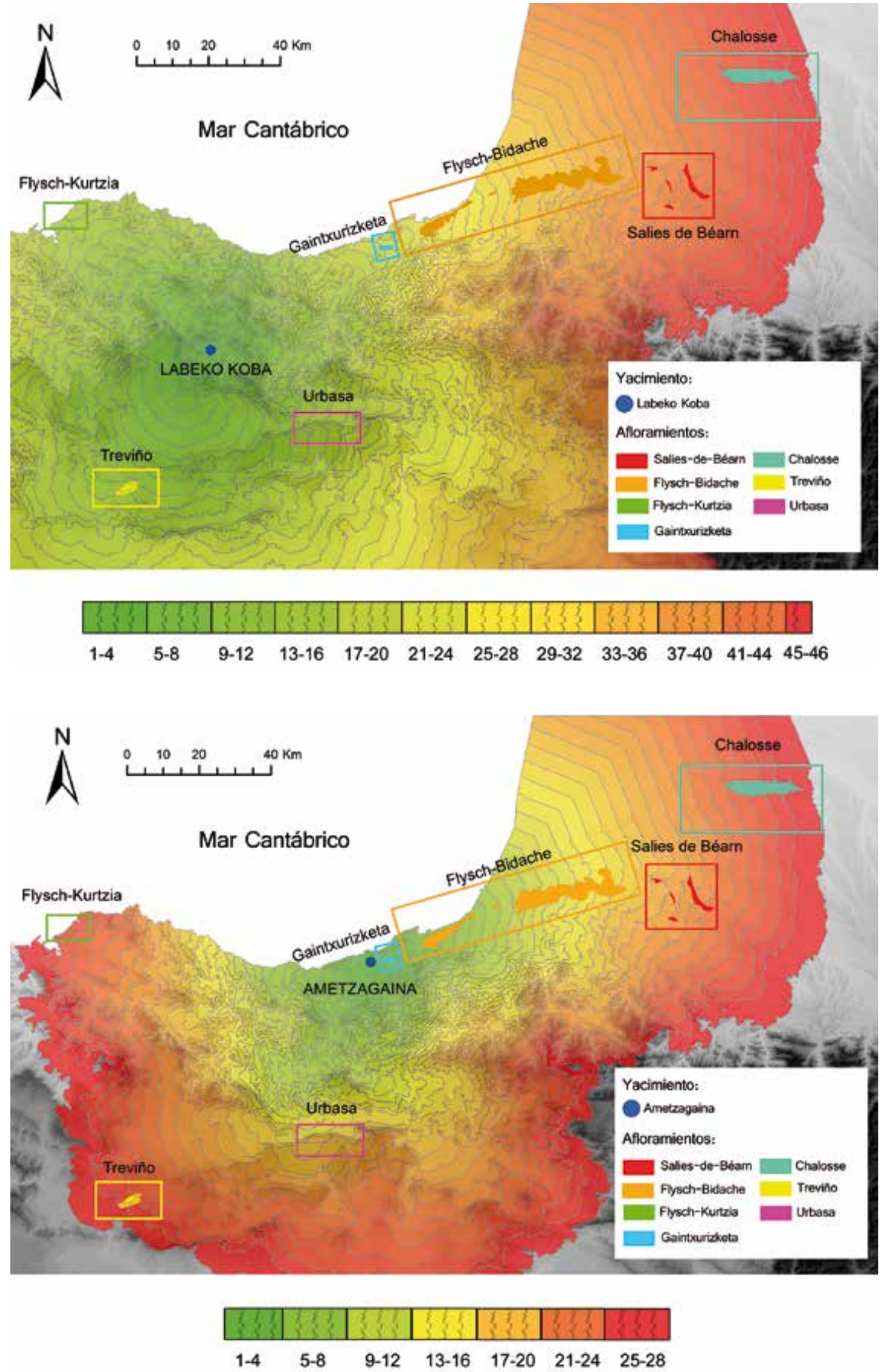

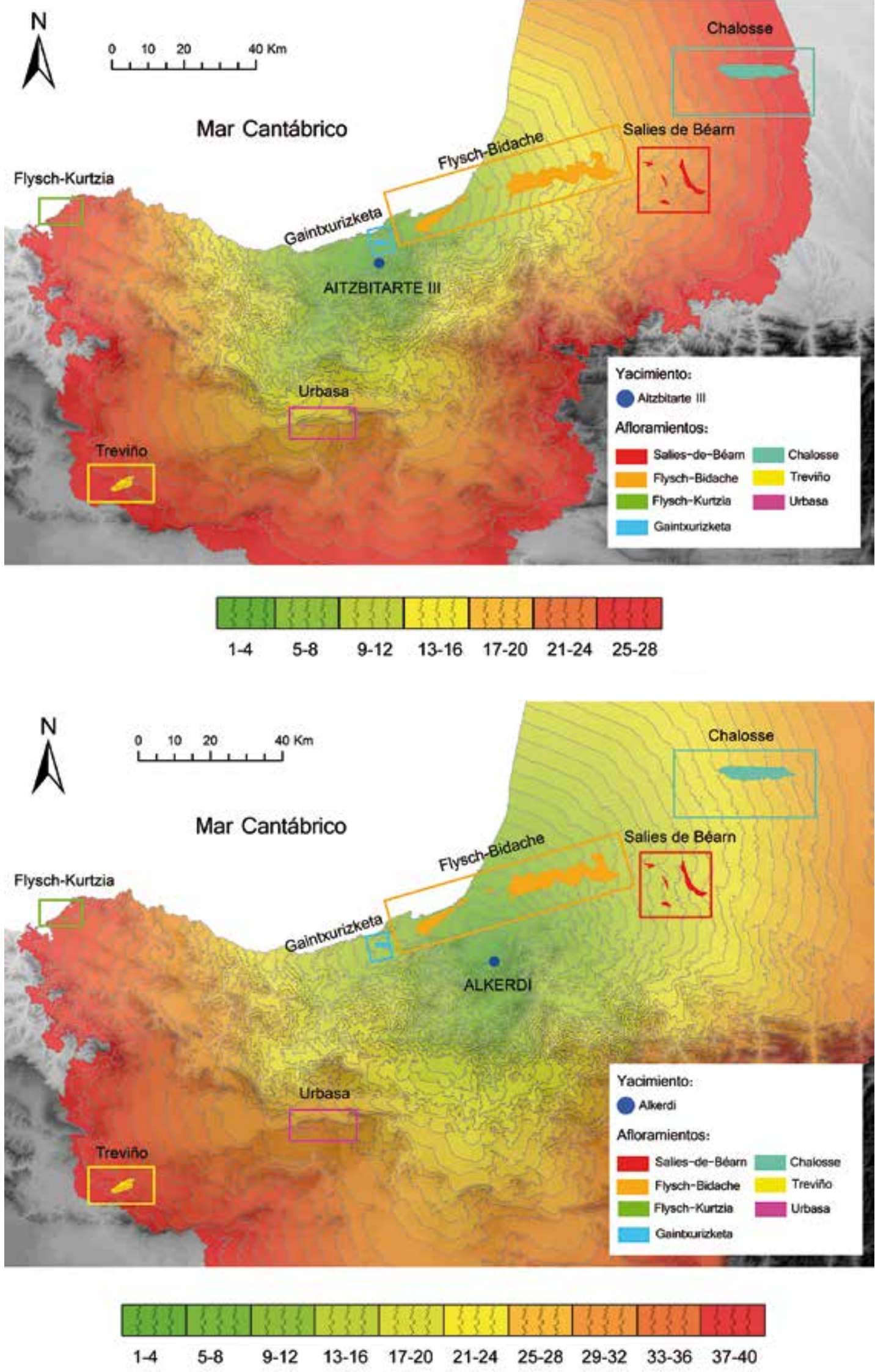

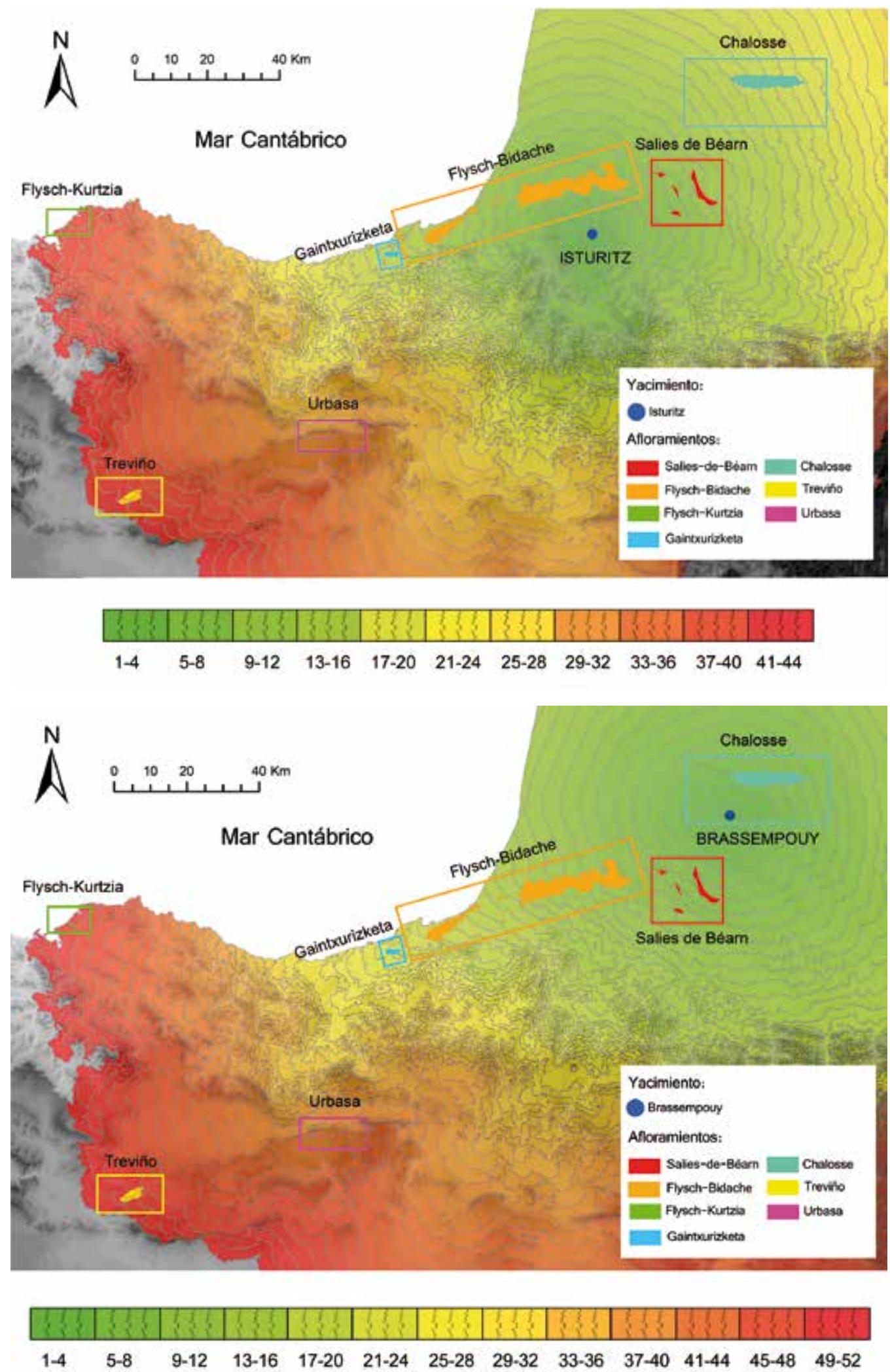

Anexos. Mapas de costes que muestran el esfuerzo que implica el desplazamiento desde cada uno de los yacimientos a los diferentes afloramientos. Appendix. Cost surface maps showing the cost of traveling from each site to the different flint outcrops. 